Interfaces and Free Boundaries 12 (2010), 251-277

DOI $10.4171 / \mathrm{IFB} / 234$

\title{
On the existence of mean curvature flow with transport term
}

\author{
CHUN LIU \\ IMA, University of Minnesota, Minneapolis, MN 55455-0134, USA \\ E-mail: liu@ima.umn.edu \\ NORIFUMI SATO \\ Furano H.S., Furano, Hokkaido 076-0011, Japan \\ E-mail: differ@mb.infosnow.ne.jp \\ Yoshiniro TONEGAWA \\ Department of Mathematics, Hokkaido University, Sapporo 060-0810, Japan \\ E-mail: tonegawa@math.sci.hokudai.ac.jp
}

[Received 27 October 2009]

\begin{abstract}
We prove the global-in-time existence of weak solution for a hypersurface evolution problem where the velocity is the sum of the mean curvature and arbitrarily given non-smooth vector field in a suitable Sobolev space. The approximate solution is obtained by the Allen-Cahn equation with transport term. By establishing the density ratio upper bound on the phase boundary measure it is shown that the limiting surface moves with the desired velocity in the sense of Brakke.
\end{abstract}

2010 Mathematics Subject Classification: Primary 53C44; Secondary 35K55, 49Q15.

Keywords: Mean curvature flow; varifold; Allen-Cahn equation; phase field method.

\section{Introduction}

In this paper we establish the existence of a family $\{\Gamma(t)\}_{0<t<\infty}$ of hypersurfaces whose velocity is

$$
V_{\Gamma}=(u \cdot n) n+H \quad \text { on } \Gamma(t), \quad t \geqslant 0 .
$$

Here $n$ is the unit normal vector and $H$ is the mean curvature vector of $\Gamma(t)$, respectively. A domain $\Omega^{+}(0) \subset \Omega=\mathbb{T}^{d}=(\mathbb{R} / \mathbb{Z})^{d}$ with boundary $\Gamma(0)$ and a vector field $u$ which depends both on $x$ and $t$ are given. The main result of the present paper is that there exists an integral varifold solution for 11.1 as long as the given transport term $u$ belongs to $L_{\mathrm{loc}}^{p}\left([0, \infty) ;\left(W^{1, p}(\Omega)\right)^{d}\right)$ for $p>(d+2) / 2$ and $d=2,3$ (see Theorem 2.2 for the precise statement). The result is a natural generalization of the pioneering work by Brakke [5] who proved the existence of integral varifolds moving by mean curvature $(u=0)$. Various notions of weak solutions for motion by mean curvature have since been developed along with approximation schemes such as viscosity solutions via the level set method $([4,6,11])$, minimizing movements method $([3,17])$ and phase field method ([14]) to name but a few. As for local regularity of weak solutions, we mention the works by Brakke [5], White [28, 29] and Ecker [8, 9].

Motivations to consider (1.1) are (i) to understand the range of perturbations within which we may obtain the integral varifold solutions and (ii) to obtain results applicable to coupled problems which typically set $u$ in a Sobolev space. The latter problems include the two-phase fluid flow problem considered in [16, 18] where $u$ satisfies a coupled Navier-Stokes equation. For that problem 
we cannot expect a good global-in-time regularity of $u$ such as Lipschitz in the space variables. We note that the natural class for the level set method for problem (1.1) is for $u$ to be in the Lipschitz class in the space variables [4]. An application to the two-phase flow problems will appear in a separate paper.

For the construction of the weak solution, we utilize the phase field method via an equation similar to the Allen-Cahn equation [2],

$$
\frac{\partial \varphi}{\partial t}=\Delta \varphi-\frac{W^{\prime}(\varphi)}{\varepsilon^{2}} .
$$

Here $W$ is the equal depth double-well potential and we set $W(\varphi)=\left(1-\varphi^{2}\right)^{2} / 2$. When $\varepsilon \rightarrow 0$ and with an appropriate set of initial data at $t=0$, the solution $\varphi$ is approximately \pm 1 on a bulk region with a thin interface region of thickness $O(\varepsilon)$. It has been proved by many authors under various assumptions that the limit interface evolves by mean curvature. In the setting of geometric measure theory, Ilmanen [14] proved that the limit surface measures $\mu_{t}$ of (AC) are rectifiable and satisfy the mean curvature flow equation in the sense of Brakke. The third author [27] proved that $\mu_{t}$ is integral (see Sec. 2.2) so that the limit measures obtained as the limit of (AC) have all the measure-theoretic properties satisfied by the varifold solutions constructed by Brakke in [5]. For $d=2,3$, the second author [24] noticed that one can give a very short and unified proof of [14] and [27] by utilizing the results by Röger and Schätzle [23]. We use the latter method in the present paper instead of that of Ilmanen. The main point of the present paper is the analysis of the Allen-Cahn equation with transport term

$$
\frac{\partial \varphi}{\partial t}+u \cdot \nabla \varphi=\Delta \varphi-\frac{W^{\prime}(\varphi)}{\varepsilon^{2}}
$$

which is expected to approximate the motion law (1.1). We point out that we need a certain growth rate control of $\|u\|_{L^{\infty}(\Omega \times[0, T])}=o\left(\varepsilon^{-1 / 2}\right)$ for the approximation scheme in order to tame the transport effect. Though such bound may not be sharp, some type of control in terms of the power of $\varepsilon$ seems necessary to preserve the $O(\varepsilon)$-scale phase boundary profile of hyperbolic tangent. The key technical point is the claim that the upper density ratio of the surface measure is uniformly bounded (see Theorem 3.1) under the natural assumptions in our setting. It roughly says that the regularizing effect of mean curvature flow 'wins' over the transport effect as long as $u$ retains the regularity specified in Theorem 3.1. In proving this we utilize the monotonicity formula due to Huisken [12] and Chen-Struwe [7], which again was first used in the context of the Allen-Cahn equation by Ilmanen [14]. In this part of the proof $p>(d+2) / 2$ is essential but $d$ does not need to be 2,3 .

Regarding the approximation scheme via the Allen-Cahn equation and its derivatives, there have been a colossal amount of related work. Thus we restrict the references to the ones with geometric measure theory settings and sufficiently close technical relevance. Soner [26] considered the phase field equation similar to $(1.2)$ coupled with a nonlinear parabolic equation. The system is equipped with a natural energy dissipation law and a candidate for a Chen-Struwe-Huiskentype monotonicity formula. The main thrust of [26] is to prove that the so-called discrepancy measure vanishes in the limit, which is similar to our concern in the present paper. Mugnai-Röger introduced the notion of $L^{2}$-flow [20] which was developed to describe an evolution of integral varifolds with $L^{2}$ mean curvature and $L^{2}$ generalized velocity. They subsequently studied a wide range of surface evolution problems including [1.2] in [21] and derived that the limit measure is $L^{2}$-flow. The notion of $L^{2}$-flow can characterize a very wide class of surface evolution problems with little regularity requirement for the velocity, typically under the assumption or a priori estimate 
that $L_{\text {loc }}^{2}\left([0, \infty) ;\left(L^{\infty}(\Omega)\right)^{d}\right)$ is bounded. The results of the present paper have some intersections in this regard, particularly for $n=2$, but are different from [21] for $n=3$.

The paper is organized as follows. In Section 2 we set out the basic definitions and explain the main theorem. Section 3 concerns the main technical proof of the upper density ratio bound 3.8]. In Section 4 we characterize the limit measures of surface energies, and Section 5 completes the proof of the main existence theorem. In Section 6 we make final remarks.

\section{Preliminaries and main results}

\subsection{Basic notations}

For $A, B \in \mathbb{R}^{d^{2}}$ we denote $A: B=\sum A_{i j} B_{i j}$ and $|A|=\sqrt{A: A}$. For $a \in \mathbb{R}^{d}, a \otimes a$ is the matrix with the entries $a_{i} a_{j}, i, j=1, \ldots, d$. We write $B_{r}(x)=\{y|| x-y \mid<r\}$ and $\omega_{k}$ is the $k$ dimensional volume of the unit ball in $\mathbb{R}^{k}$. We write $L^{p}(\Omega)$ for the space of $p$-th power integrable functions and $W^{1, p}(\Omega)=\left\{u \in L^{p}(\Omega) \mid \nabla u \in\left(L^{p}(\Omega)\right)^{d}\right\}$. $\left(L^{p}(\Omega)\right)^{d}$ is the $d$-vector valued $L^{p}(\Omega)$ functions and similarly for $\left(W^{1, p}(\Omega)\right)^{d}$. For a Radon measure $\mu, L^{p}(\mu)$ is the space of $p$-th power integrable functions with respect to $\mu$. We write the space of bounded variation functions as $B V(\Omega)$. We denote by $\chi_{A}$ the characteristic function of a set $A$. For the standard notions related to $B V$-functions such as sets of finite perimeter and reduced boundaries we refer the readers to [10]. Finally $\mathcal{H}^{k}$ is the $k$-dimensional Hausdorff measure and $\mathcal{L}^{d}$ is the Lebesgue measure in $\mathbb{R}^{d}$.

\subsection{Varifold notations}

We recall some notions from geometric measure theory and refer to [1, 5, 25] for more details. A general $k$-varifold in $\mathbb{R}^{d}$ is a Radon measure on $\mathbb{R}^{d} \times G_{k}\left(\mathbb{R}^{d}\right)$, where $G_{k}\left(\mathbb{R}^{d}\right)$ is the Grassmann manifold of unoriented $k$-dimensional subspaces in $\mathbb{R}^{d}$. We denote the set of all general $k$-varifolds by $\mathbf{V}_{k}\left(\mathbb{R}^{d}\right)$. When $S$ is a $k$-dimensional subspace, we also use $S$ to denote the $d$ by $d$ matrix representing the orthogonal projection $\mathbb{R}^{d} \rightarrow S$. The first variation of $V$ can be written as

$\delta V(g)=\int_{\mathbb{R}^{d} \times G_{k}\left(\mathbb{R}^{d}\right)} \nabla g(x): S \mathrm{~d} V(x, S)=-\int_{\mathbb{R}^{d}} g(x) \cdot H(x) \mathrm{d}\|V\|(x) \quad$ if $\|\delta V\| \ll\|V\|$.

Here $V \in \mathbf{V}_{k}\left(\mathbb{R}^{d}\right),\|V\|$ is the mass measure of $V, g \in\left(C_{c}^{1}\left(\mathbb{R}^{d}\right)\right)^{d}, H=H_{V}$ is the generalized mean curvature vector if it exists and $\|\delta V\| \ll\|V\|$ denotes that $\|\delta V\|$ is absolutely continuous with respect to $\|V\|$.

We call a Radon measure $\mu$-integral if it is represented as $\mu=\theta \mathcal{H}^{k}\left\lfloor X\right.$, that is, $\int_{\mathbb{R}^{d}} \phi \mathrm{d} \mu=$ $\int_{X} \phi \theta \mathrm{d} \mathcal{H}^{k}$ for $\phi \in C_{c}\left(\mathbb{R}^{k}\right)$. Here $X$ is a countably $k$-rectifiable, $\mathcal{H}^{k}$-measurable set, and $\theta \in$ $L_{\text {loc }}^{1}\left(\mathcal{H}^{k}\left\lfloor_{X}\right)\right.$ is positive and integer-valued $\mathcal{H}^{k}$ a.e on $X$. When $\theta=1$ a.e. on $X$, we say $\mu$ has unit density. We denote the set of all $k$-integral Radon measures by $\mathcal{I} \mathcal{M}_{k}\left(\mathbb{R}^{d}\right)$. To each such $\mu$ corresponds a unique $k$-varifold $V$ defined by

$$
\int_{\mathbb{R}^{d} \times G_{k}\left(\mathbb{R}^{d}\right)} \phi(x, S) \mathrm{d} V(x, S)=\int_{\mathbb{R}^{d}} \phi\left(x, T_{x} \mu\right) \mathrm{d} \mu(x) \quad \text { for } \phi \in C_{c}\left(\mathbb{R}^{d} \times G_{k}\left(\mathbb{R}^{d}\right)\right),
$$

where $T_{x} \mu$ is the approximate tangent $k$-plane of $X$ at $x$ which exists $\mathcal{H}^{k}$ a.e on $X$. Note that $\mu=\|V\|$ under this correspondence. We make this identification in the following. For this reason we define $H_{\mu}$ as $H_{V}$ (or simply $H$ ) if the latter exists. When $X$ is a $C^{2}$ submanifold without 
boundary and $\theta$ is constant on $X, H$ corresponds to the usual mean curvature vector of $X$. In the following we suitably adapt the above notions to $\Omega=\mathbb{T}^{d}$, such as $\mathbf{V}_{k}(\Omega)$ and $\mathcal{I} \mathcal{M}_{k}(\Omega)$, which presents no essential difficulties.

\subsection{Weak formulation of velocity}

For a sufficiently smooth surface $\Gamma(t)$ moving by the velocity $(1.1)$, the following holds for any $\phi \in C^{2}\left(\Omega ; \mathbb{R}^{+}\right)$due to the first variation formula 2.1 :

$$
\frac{\mathrm{d}}{\mathrm{d} t} \int_{\Gamma(t)} \phi \mathrm{d} \mathcal{H}^{d-1} \leqslant \int_{\Gamma(t)}(-\phi H+\nabla \phi) \cdot\{H+(u \cdot n) n\} \mathrm{d} \mathcal{H}^{d-1} .
$$

One can check that having this inequality for any $\phi \in C^{2}\left(\Omega ; \mathbb{R}^{+}\right)$implies 1.1 , thus 2.2 is equivalent to (1.1). Without the transport term $u$ this is Brakke's approach to the mean curvature flow. To define $u$ as the trace on $\Gamma(t)$ we need the following

THEOREM 2.1 (Meyers-Ziemer inequality) For a Radon measure $\mu$ on $\mathbb{R}^{d}$ with $D=$ $\sup _{r>0, x \in \mathbb{R}^{d}} \mu\left(B_{r}(x)\right) /\left(\omega_{d-1} r^{d-1}\right)$ and $1 \leqslant q<d$,

$$
\int_{\mathbb{R}^{d}}|\phi|^{\frac{q(d-1)}{d-q}} \mathrm{~d} \mu \leqslant c_{M Z} D\left(\int_{\mathbb{R}^{d}}|\nabla \phi|^{q} \mathrm{~d} x\right)^{\frac{d-1}{d-q}}
$$

for $\phi \in C_{c}^{1}\left(\mathbb{R}^{d}\right)$. Here $c_{M Z}=c_{M Z}(d, q)$.

See [19] and [30, p. 266] for $q=1$. The statement for $1 \leqslant q<d$ is easily derived by the Hölder inequality and will be used later. By localizing Theorem 2.1 to $\Omega=\mathbb{T}^{d}$ and choosing $q=1$ we obtain (with $D=\sup _{1 / 2>r>0, x \in \Omega} \mu\left(B_{r}(x)\right) /\left(\omega_{d-1} r^{d-1}\right)$ )

$$
\int_{\Omega}|\phi|^{2} \mathrm{~d} \mu \leqslant c_{M Z} D\|\phi\|_{L^{2}(\Omega)}\|\nabla \phi\|_{L^{2}(\Omega)},
$$

where $c_{M Z}$ may differ from 2.3 and only depends on $d$. The inequality allows us to define $\int_{\Omega}|\phi|^{2} \mathrm{~d} \mu$ for $\phi \in W^{1,2}(\Omega)$ by the standard density argument.

We define, for any Radon measure $\mu, u \in\left(W^{1,2}(\Omega)\right)^{d}$ and $\phi \in C^{2}\left(\Omega ; \mathbb{R}^{+}\right)$,

$$
\mathcal{B}(\mu, u, \phi)=\int_{\Omega}(-\phi H+\nabla \phi) \cdot\{H+(u \cdot n) n\} \mathrm{d} \mu
$$

if $\mu \in \mathcal{I M}_{d-1}(\Omega)$ with $H \in\left(L^{2}(\mu)\right)^{d}$ and $\sup _{1 / 2>r>0, x \in \Omega} \mu\left(B_{r}(x)\right) /\left(\omega_{d-1} r^{d-1}\right)<\infty$. This gives a well-defined finite value due to the stated conditions and (2.4). If any one of the conditions is not satisfied, we define $\mathcal{B}(\mu, u, \phi)=-\infty$.

\subsection{Main existence results}

Our main results are the following.

TheOREM 2.2 Suppose $d=2$ or $3, p>(d+2) / 2$ and $\Omega=\mathbb{T}^{d}$. Given any

$$
u \in L_{\mathrm{loc}}^{p}\left([0, \infty) ;\left(W^{1, p}(\Omega)\right)^{d}\right)
$$

and $\Omega^{+}(0) \subset \Omega$ having $C^{1}$ boundary $\Gamma(0)$, there exist 
(i) a family $\left\{\mu_{t}\right\}_{t \in[0, \infty)}$ of Radon measures with $\mu_{t} \in \mathcal{I M}_{d-1}(\Omega)$ for a.e. $t \in[0, \infty)$ and

(ii) $\varphi \in B V_{\mathrm{loc}}(\Omega \times[0, \infty)) \cap L_{\mathrm{loc}}^{\infty}([0, \infty) ; B V(\Omega)) \cap C_{\mathrm{loc}}^{1 / 2}\left([0, \infty) ; L^{1}(\Omega)\right)$

such that the following hold:

(a) For all $0 \leqslant t_{1}<t_{2}<\infty$ and $\phi \in C^{2}\left(\Omega ; \mathbb{R}^{+}\right)$we have

$$
\mu_{t_{2}}(\phi)-\mu_{t_{1}}(\phi) \leqslant \int_{t_{1}}^{t_{2}} \mathcal{B}\left(\mu_{t}, u(\cdot, t), \phi\right) \mathrm{d} t .
$$

Moreover $\mathcal{B}\left(\mu_{t}, u(\cdot, t), \phi\right) \in L_{\text {loc }}^{1}([0, \infty))$ and $\mu_{0}=\mathcal{H}^{d-1} \iota_{\Gamma}(0)$.

(b) The function $\varphi$ satisfies the following properties.

(1) $\varphi(\cdot, t)= \pm 1$ a.e. on $\Omega$ and for all $t \in[0, \infty)$,

(2) $\varphi(\cdot, 0)=\chi_{\Omega^{+}(0)}-\chi_{\Omega \backslash \Omega^{+}(0)}$ a.e. on $\Omega$,

(3) $\operatorname{spt}|\nabla \chi\{\varphi(\cdot, t)=1\}| \subset \operatorname{spt} \mu_{t}$ for all $t \in[0, \infty)$.

(c) There exists $T_{1}=T_{1}\left(\|u\|_{L^{p}\left([0,1] ;\left(W^{1, p}(\Omega)\right)^{d}\right)}, \Omega^{+}(0), p\right) \leqslant 1$ such that $\mu_{t}$ has unit density for a.e. $t \in\left[0, T_{1}\right]$. In addition $\left|\nabla \chi_{\{\varphi=1\}}\right|=\mu_{t}$ for a.e. $t \in\left[0, T_{1}\right]$.

The inequality (2.6) shows that $\mu_{t}$ satisfies the desired motion law in the sense stated in (2.2) in the integral form. Define $\Gamma(t)$ by the relation $\mu_{t}=\theta \mathcal{H}^{d-1}\left\lfloor\Gamma(t)\right.$ whenever $\mu_{t} \in \mathcal{I M}_{d-1}(\Omega)$. It is appropriate to say that $\{\Gamma(t)\}_{t \geqslant 0}$ (with multiplicity $\theta$ ) moves by the velocity $[1.1$ in the sense of Brakke. In addition we have

THEOREM 2.3 The family $\left\{\mu_{t}\right\}_{t \in[0, \infty)}$ of Radon measures obtained in Theorem 2.2 satisfies the following properties.

(a) For all $0 \leqslant t_{1}<t_{2}<\infty$ and $\phi \in C^{3}\left(\Omega \times[0, \infty)\right.$; $\left.\mathbb{R}^{+}\right)$we have

$$
\mu_{t_{2}}\left(\phi\left(\cdot, t_{2}\right)\right)-\mu_{t_{1}}\left(\phi\left(\cdot, t_{1}\right)\right) \leqslant \int_{t_{1}}^{t_{2}}\left(\left(\int_{\Omega} \frac{\partial \phi}{\partial t}(\cdot, t) \mathrm{d} \mu_{t}\right)+\mathcal{B}\left(\mu_{t}, u(\cdot, t), \phi(\cdot, t)\right)\right) \mathrm{d} t .
$$

(b) $\sup _{t \in[0, T], x \in \Omega, 0<r<1 / 2} \mu_{t}\left(B_{r}(x)\right) /\left(\omega_{d-1} r^{d-1}\right)<\infty$ for all $T>0$.

The property (a) may be useful to establish the partial regularity ([5, Sec. 3.5]).

REMARK 2.4 The difficulty of multiplicities have been often encountered in the measure-theoretic setting like ours. Varifold solutions constructed by Brakke [5] have the same properties in this regard. On the other hand, (c) says that there is no 'folding' for some time interval [0, $\left.T_{1}\right]$.

REMARK 2.5 The initial data $\Gamma(0)=\partial \Omega^{+}(0)$ can be less regular than $C^{1}$. The same statements of Theorems 2.2 and 2.3 except for (c) hold for $\Gamma(0)$ with the following properties: we assume $\mathcal{H}^{d-1}(\Gamma(0))<\infty$ and that there exists a sequence of domains $\Omega^{+}(0)^{i}$ with $C^{3}$ boundary $\Gamma(0)^{i}$ such that

$$
\begin{aligned}
& \sup _{i, 0<r \leqslant 1 / 2, x \in \Omega} \frac{\mathcal{H}^{d-1}\left(B_{r}(x) \cap \Gamma(0)^{i}\right)}{\omega_{d-1} r^{d-1}}<\infty, \\
& \lim _{i \rightarrow \infty} \mathcal{L}^{d}\left(\Omega^{+}(0) \triangle \Omega^{+}(0)^{i}\right)=0, \\
& \lim _{i \rightarrow \infty}\left|\nabla \chi_{\Omega^{+}(0)^{i}}\right|=\left|\nabla \chi_{\Omega^{+}(0)}\right| \quad \text { as measures. }
\end{aligned}
$$


For (c) to be true we additionally require that

$$
\sup _{0<r<r_{0}, x \in \Omega} \frac{\left|\nabla \chi_{\Omega^{+}(0)}\right|\left(B_{r}(x)\right)}{\omega_{d-1} r^{d-1}} \leqslant 1+o\left(r_{0}\right) .
$$

By a suitable application of the implicit function theorem, partition of unity and smoothing, one can show that $C^{1}$ boundary satisfies all of the above conditions. They are also satisfied by surfaces with irregular thin spikes, which are not $C^{1}$, for example. The proof is given for $\Gamma(0)$ with the above conditions. On the other hand it is clear that the above conditions exclude finite perimeter sets whose measure-theoretic boundaries have infinite density points.

\subsection{Theorem to be used}

We use the following theorem in a similar way to [24]:

THEOREM 2.6 (Röger and Schätzle [23]) Suppose $d=2$ or 3 and $\Omega \subset \mathbb{R}^{d}$ is an open set. Let $\varphi^{\varepsilon} \in C^{2}(\Omega)$ and set

$$
\mu^{\varepsilon}=\frac{1}{\sigma}\left(\frac{\varepsilon\left|\nabla \varphi^{\varepsilon}\right|^{2}}{2}+\frac{W\left(\varphi^{\varepsilon}\right)}{\varepsilon}\right) \mathrm{d} x, \quad \alpha^{\varepsilon}=\frac{\varepsilon}{\sigma}\left(\Delta \varphi^{\varepsilon}-\frac{W^{\prime}\left(\varphi^{\varepsilon}\right)}{\varepsilon^{2}}\right)^{2} \mathrm{~d} x
$$

where $\sigma=\int_{-1}^{1} \sqrt{2 W(s)} \mathrm{d} s$. Assume $\lim _{i \rightarrow \infty} \varepsilon_{i}=0$ and $\varphi^{\varepsilon_{i}}$ satisfies

$$
\liminf _{i \rightarrow \infty}\left(\mu^{\varepsilon_{i}}(\Omega)+\alpha^{\varepsilon_{i}}(\Omega)\right)<\infty, \quad \lim _{i \rightarrow \infty} \mu^{\varepsilon_{i}}=\mu \quad \text { as measures. }
$$

Then:

(i) $\mu \in \mathcal{I} \mathcal{M}_{d-1}(\Omega)$.

(ii) $\int_{\Omega}|H|^{2} \mathrm{~d} \mu \leqslant \liminf _{i \rightarrow \infty} \alpha^{\varepsilon_{i}}$, where $H$ is the generalized mean curvature of $\mu$.

\section{Density ratio upper bound for interface energy}

In this section we prove that the diffused interface energy satisfies a certain uniform upper density ratio bound which is crucial in the subsequent limiting procedures when $\varepsilon \rightarrow 0$. Since the estimate is of independent interest and is true for all dimensions, we state the assumptions in the form independent of the existence results in the following sections.

\subsection{Main Theorem on the upper density ratio}

TheOREM 3.1 Suppose $d \geqslant 2, \Omega=\mathbb{T}^{d}, p>(d+2) / 2, T>0,1 / 2>\gamma \geqslant 0,1 \geqslant \varepsilon>0$ and $\varphi$ satisfies

$$
\begin{aligned}
\frac{\partial \varphi}{\partial t}+u \cdot \nabla \varphi & =\Delta \varphi-\frac{W^{\prime}(\varphi)}{\varepsilon^{2}} & & \text { on } \Omega \times[0, T], \\
\varphi(x, 0) & =\varphi_{0}(x) & & \text { on } \Omega,
\end{aligned}
$$


where $\nabla^{i} u, \nabla^{j} \varphi, \nabla^{k} \varphi_{t} \in C(\Omega \times[0, T])$ for $i, k \in\{0,1\}$ and $j \in\{0,1,2,3\}$. Let $\mu_{t}$ be the Radon measure on $\Omega$ defined by

$$
\int_{\Omega} \phi(x) \mathrm{d} \mu_{t}(x)=\frac{1}{\sigma} \int_{\Omega} \phi(x)\left(\frac{\varepsilon|\nabla \varphi(x, t)|^{2}}{2}+\frac{W(\varphi(x, t))}{\varepsilon}\right) \mathrm{d} x
$$

for $\phi \in C(\Omega)$, where $\sigma=\int_{-1}^{1} \sqrt{2 W(s)} \mathrm{d} s$. Also assume that

$$
\begin{aligned}
\sup _{\Omega}\left|\varphi_{0}\right| \leqslant 1 & \text { and } \quad \sup _{\Omega} \varepsilon^{i}\left|\nabla^{i} \varphi_{0}\right| \leqslant c_{1} \quad \text { for } i \in\{1,2,3\}, \\
& \sup _{\Omega}\left(\frac{\varepsilon\left|\nabla \varphi_{0}\right|^{2}}{2}-\frac{W\left(\varphi_{0}\right)}{\varepsilon}\right) \leqslant \varepsilon^{-\gamma}, \\
& \sup _{\Omega \times[0, T]}\left\{\varepsilon^{\gamma}|u|, \varepsilon^{1+\gamma}|\nabla u|\right\} \leqslant c_{2}, \\
& \|u\|_{L^{p}\left([0, T] ;\left(W^{1, p}(\Omega)\right)^{d}\right)} \leqslant c_{3} .
\end{aligned}
$$

For $t \in[0, T]$ define

$$
D(t)=\max \left\{\sup _{x \in \Omega, 0<r \leqslant 1 / 2} \frac{1}{\omega_{d-1} r^{d-1}} \mu_{t}\left(B_{r}(x)\right), 1\right\}, \quad \text { and assume } \quad D(0) \leqslant D_{0} .
$$

Then there exist $c_{4}$ which depends only on $c_{3}, d, p, D_{0}$ and $T$, and $\epsilon_{1}>0$ which depends only on $d, p, W, c_{1}, c_{2}, c_{3}, D_{0}, \gamma$ and $T$ such that for all $0<\varepsilon \leqslant \epsilon_{1}$ and $t \in[0, T]$,

$$
D(t) \leqslant c_{4}
$$

\subsection{Monotonicity formula}

First we derive the monotonicity formula for the surface energy $\mu_{t}$ which is analogous to [14]. One essential difficulty is the control of the so-called discrepancy measure $\varepsilon|\nabla \varphi|^{2} / 2-W / \varepsilon$ and our analysis concentrates mostly on this issue.

We fix $(y, s) \in \mathbb{R}^{d} \times[0, \infty)$ and define, for any $(x, t) \in \mathbb{R}^{d} \times[0, s)$,

$$
\rho_{(y, s)}(x, t)=\frac{1}{(4 \pi(s-t))^{(d-1) / 2}} e^{-\frac{|x-y|^{2}}{4(s-t)}} .
$$

To localize the formula to $\Omega$, we fix a radially symmetric cut-off function $\eta \in C_{c}^{\infty}\left(B_{1 / 2}(0)\right)$ with $\eta=1$ on $B_{1 / 4}(0)$ and $0 \leqslant \eta \leqslant 1$. We then define

$$
\tilde{\rho}_{(y, s)}(x, t)=\rho_{(y, s)}(x, t) \eta(x-y)=\frac{1}{(4 \pi(s-t))^{(d-1) / 2}} e^{-\frac{|x-y|^{2}}{4(s-t)}} \eta(x-y) .
$$

The following can be obtained by similar computations to [14], the only difference being the extra term $u \cdot \nabla \varphi$ and the cut-off function. 
Proposition 3.2 There exist constants $c_{5}, c_{6}>0$ depending only on $d$ such that for $y \in \Omega$, $0<t<s<\infty$ and $t \leqslant T$,

$$
\begin{aligned}
\frac{\mathrm{d}}{\mathrm{d} t} \int_{\Omega} \tilde{\rho} \mathrm{d} \mu_{t}(x) \leqslant & \int_{\Omega} \tilde{\rho}|u|^{2} \mathrm{~d} \mu_{t}(x)+\frac{1}{2(s-t) \sigma} \int_{\Omega}\left(\frac{\varepsilon|\nabla \varphi|^{2}}{2}-\frac{W(\varphi)}{\varepsilon}\right) \tilde{\rho} \mathrm{d} x \\
& +c_{5} e^{-c_{6} /(s-t)}\left(\mu_{t}(\Omega)+\frac{1}{\sigma} \int_{\Omega} \varepsilon\left(\Delta \varphi-\frac{W^{\prime}(\varphi)}{\varepsilon^{2}}\right)^{2} \mathrm{~d} x\right) .
\end{aligned}
$$

Note that $\tilde{\rho}=\tilde{\rho}_{(y, s)}(x, t)$ is integrated with respect to $x$.

Proof. We present the computations on $\mathbb{R}^{d}$ and without the cut-off function $\eta$ for simplicity. In the following we write $\rho$ for $\rho_{(y, s)}(x, t)$ and set

$$
E=\frac{\varepsilon|\nabla \varphi|^{2}}{2}+\frac{W(\varphi)}{\varepsilon}, \quad L=\varphi_{t}+u \cdot \nabla \varphi=\Delta \varphi-\frac{W^{\prime}(\varphi)}{\varepsilon^{2}} .
$$

We calculate by integration by parts and completing the square:

$$
\begin{aligned}
\frac{\mathrm{d}}{\mathrm{d} t} \int_{\mathbb{R}^{d}} E \rho \mathrm{d} x= & \int_{\mathbb{R}^{d}}\left\{E \rho_{t}-\varepsilon \varphi_{t}\left(\nabla \rho \cdot \nabla \varphi+\rho \Delta \varphi-\rho \frac{W^{\prime}(\varphi)}{\varepsilon^{2}}\right)\right\} \mathrm{d} x \\
= & \int_{\mathbb{R}^{d}}\left\{E \rho_{t}-\varepsilon(L-u \cdot \nabla \varphi)(\nabla \rho \cdot \nabla \varphi+\rho L)\right\} \mathrm{d} x \\
= & \int_{\mathbb{R}^{d}}\left\{E \rho_{t}-\varepsilon \rho\left(L^{2}+L \frac{\nabla \rho \cdot \nabla \varphi}{\rho}\right)+\varepsilon \rho u \cdot \nabla \varphi\left(L+\frac{\nabla \rho \cdot \nabla \varphi}{\rho}\right)\right\} \mathrm{d} x \\
= & \int_{\mathbb{R}^{d}}\left\{E \rho_{t}-\varepsilon \rho\left(L+\frac{\nabla \rho \cdot \nabla \varphi}{\rho}\right)^{2}+\varepsilon\left(L \nabla \rho \cdot \nabla \varphi+\frac{(\nabla \rho \cdot \nabla \varphi)^{2}}{\rho}\right)\right. \\
& \left.+\varepsilon \rho u \cdot \nabla \varphi\left(L+\frac{\nabla \rho \cdot \nabla \varphi}{\rho}\right)\right\} \mathrm{d} x \\
\leqslant & \int_{\mathbb{R}^{d}}\left\{E \rho_{t}+\varepsilon\left(L \nabla \rho \cdot \nabla \varphi+\frac{(\nabla \rho \cdot \nabla \varphi)^{2}}{\rho}\right)+\frac{1}{4} \varepsilon \rho(u \cdot \nabla \varphi)^{2}\right\} \mathrm{d} x .
\end{aligned}
$$

By integration by parts we have

$$
\int_{\mathbb{R}^{d}} \varepsilon L \nabla \rho \cdot \nabla \varphi \mathrm{d} x=\int_{\mathbb{R}^{d}}\left(-\varepsilon \nabla \varphi \otimes \nabla \varphi: \nabla^{2} \rho+E \Delta \rho\right) \mathrm{d} x .
$$

Two important identities which $\rho$ satisfies are

$$
\rho_{t}+\Delta \rho=-\frac{\rho}{2(s-t)}, \quad \rho_{t}+\Delta \rho-\frac{\nabla \varphi \otimes \nabla \varphi}{|\nabla \varphi|^{2}}: \nabla^{2} \rho+\frac{(\nabla \rho \cdot \nabla \varphi)^{2}}{\rho|\nabla \varphi|^{2}}=0,
$$

and substituting 3.11 and 3.12 into 3.10, we obtain 3.9 without the last term. When one computes the above with $\tilde{\rho}$ instead of $\rho$, we have the additional term

$$
\int_{\mathbb{R}^{d}} \varepsilon \varphi_{t} \rho \nabla \varphi \cdot \nabla \eta \mathrm{d} x
$$


in the first integration by parts in (3.10). Deriving (3.11) we also have additional terms which can be bounded by $\sigma \mu_{t}(\Omega) c e^{-c /(s-t)}$ with a suitable constant $c>0$. For (3.13) we estimate, using (3.1),

$$
\begin{aligned}
\int_{\mathbb{R}^{d}} \varepsilon\left|\varphi_{t}\right| & |\nabla \varphi||\nabla \eta| \rho \mathrm{d} x \\
& \leqslant \frac{1}{4} \int_{|x-y| \geqslant 1 / 4} \varepsilon\left(1+|u|^{2}\right)|\nabla \varphi|^{2} \tilde{\rho} \mathrm{d} x+\int_{\mathbb{R}^{d}} \varepsilon\left(|\nabla \varphi|^{2}+\left(\Delta \varphi-\frac{W^{\prime}}{\varepsilon^{2}}\right)^{2}\right) \frac{|\nabla \eta|^{2}}{\eta} \rho \mathrm{d} x \\
& \leqslant \frac{1}{4} \int_{\mathbb{R}^{d}} \varepsilon|u|^{2}|\nabla \varphi|^{2} \tilde{\rho} \mathrm{d} x+c e^{-c /(s-t)}\left(\sigma \mu_{t}(\Omega)+\int_{\Omega} \varepsilon\left(\Delta \varphi-\frac{W^{\prime}}{\varepsilon^{2}}\right)^{2} \mathrm{~d} x\right) .
\end{aligned}
$$

Thus with suitable choices of $c_{5}$ and $c_{6}$ depending only on $d$, we obtain 3.9 .

\subsection{Estimates on $\Omega \times[0, T]$}

Here we collect estimates which hold on $\Omega \times[0, T]$ for sufficiently small $\varepsilon>0$. These are in contrast with the next subsection where we assume the density upper ratio bound $(3.8)$ for $t \in\left[0, T_{1}\right]$. First we prove the following uniform gradient bound. Even though the estimate may be well-known, we include the proof for completeness. The point here is that we want a uniform gradient estimate which is independent of the terms such as $u_{t}$.

Lemma 3.3 There exists $c_{7}$ depending only on $d, c_{1}, c_{2}$ and $W$ such that

$$
\sup _{\Omega \times[0, T]}|\nabla \varphi| \leqslant c_{7} / \varepsilon
$$

Proof. Rescale the domain by $x \mapsto x / \varepsilon$ and $t \mapsto t / \varepsilon^{2}$. Then the equation (3.1) is

$$
\varphi_{t}+\varepsilon u \cdot \nabla \varphi=\Delta \varphi-W^{\prime}(\varphi) .
$$

By the $L^{p}$ estimate of the heat kernel [15, p. 288], we have

$$
\begin{aligned}
& v_{t}-\Delta v=f \text { on } B_{2} \times[0,2] \Rightarrow \\
& \quad\left\|v_{t}, \nabla^{2} v\right\|_{L^{q}\left(B_{1} \times[j, 2]\right)} \leqslant c(d, q)\left(\|f, \nabla v, v\|_{L^{q}\left(B_{2} \times[0,2]\right)}+(1-j)\|v(\cdot, 0)\|_{W^{2, q}\left(B_{2}\right)}\right)
\end{aligned}
$$

for $j=0$ (up to $t=0$ ) or $j=1$ (interior estimate) and for $1<q<\infty$. Here we discuss the case of up to $t=0$. The interior case is similar. By multiplying 3.15 by $\phi^{2} \varphi$ where $\phi \in C_{c}^{1}\left(B_{3}\right)$ is a suitable cut-off function, and by integration by parts, we easily obtain (with $|\varphi| \leqslant 1$ and (3.5))

$$
\int_{0}^{2} \int_{B_{2}}|\nabla \varphi|^{2} \mathrm{~d} x \mathrm{~d} t \leqslant c\left(W, c_{2}\right)
$$

Thus by 3.15, 3.5, 3.3, 3.16) and 3.17,

$$
\int_{0}^{2} \int_{B_{1}}\left(\left(\varphi_{t}\right)^{2}+\left|\nabla^{2} \varphi\right|^{2}\right) \mathrm{d} x \mathrm{~d} t \leqslant c\left(d, W, c_{1}, c_{2}\right)
$$

By differentiating (3.15) with respect to $x_{i}$ we have

$$
\left(\varphi_{x_{i}}\right)_{t}-\Delta \varphi_{x_{i}}=-\varepsilon u_{x_{i}} \cdot \nabla \varphi-\varepsilon u \cdot \nabla \varphi_{x_{i}}-W^{\prime \prime}(\varphi) \varphi_{x_{i}} .
$$


By 3.16, 3.18 and 3.5 with $q=2$ applied to 3.19,

$$
\int_{0}^{2} \int_{B_{1}}\left(\left|\nabla \varphi_{t}\right|^{2}+\left|\nabla^{3} \varphi\right|^{2}\right) \mathrm{d} x \mathrm{~d} t \leqslant c\left(d, W, c_{1}, c_{2}\right) .
$$

Then we have a $W^{1,2}$-norm estimate of $\nabla \varphi$ on $B_{1} \times[0,2]$ and by the Sobolev embedding theorem,

$$
\|\nabla \varphi\|_{L^{2(d+1) /(d-1)}\left(B_{1} \times[0,2]\right)} \leqslant c\left(d, W, c_{1}, c_{2}\right) .
$$

We can use this estimate in (3.15). By (3.16) with $q=2(d+1) /(d-1)$, we obtain the improved estimates for $\varphi_{t}$ and $\nabla^{2} \varphi$, and then for $\nabla \varphi_{t}$ by (3.19). We can repeat this process until the integrability exponent $q$ exceeds $d$. Then this gives the desired estimate for $\varphi$ in terms of $d, c_{1}, c_{2}, W$.

The next lemma is proved in a similar manner to [13, Proposition 3.3].

LEMmA 3.4 There exists $\epsilon_{2}=\epsilon_{2}\left(d, W, c_{1}, c_{2}\right)>0$ such that, for $0<\varepsilon<\epsilon_{2}$,

$$
\frac{\varepsilon|\nabla \varphi|^{2}}{2}-\frac{W(\varphi)}{\varepsilon} \leqslant\left(2+8 c_{2}\right) \varepsilon^{-\gamma} \quad \text { on } \Omega \times[0, T]
$$

Proof. Rescale the domain as in Lemma 3.3 Define

$$
\xi=\frac{|\nabla \varphi|^{2}}{2}-W(\varphi)-G(\varphi)
$$

where $G$ will be chosen later. We compute $\xi_{t}+\varepsilon u \cdot \nabla \xi-\Delta \xi$ and obtain

$$
\begin{aligned}
\xi_{t}+\varepsilon u \cdot \nabla \xi-\Delta \xi= & \nabla \varphi \cdot \nabla \varphi_{t}-\left(W^{\prime}+G^{\prime}\right) \varphi_{t}+\varepsilon u \otimes \nabla \varphi: \nabla^{2} \varphi-\varepsilon\left(W^{\prime}+G^{\prime}\right) u \cdot \nabla \varphi \\
& -\left|\nabla^{2} \varphi\right|^{2}-\nabla \varphi \cdot \nabla(\Delta \varphi)+\left(W^{\prime}+G^{\prime}\right) \Delta \varphi+\left(W^{\prime \prime}+G^{\prime \prime}\right)|\nabla \varphi|^{2} .
\end{aligned}
$$

Differentiate 3.15 with respect to $x_{j}$, multiply by $\varphi_{x_{j}}$ and sum over $j$ to obtain

$$
\nabla \varphi \cdot \nabla \varphi_{t}+\varepsilon \nabla u: \nabla \varphi \otimes \nabla \varphi+\varepsilon u \otimes \nabla \varphi: \nabla^{2} \varphi=\nabla \varphi \cdot \nabla(\Delta \varphi)-W^{\prime \prime}|\nabla \varphi|^{2} .
$$

Combining 3.15, 3.23, and 3.24, we obtain

$$
\xi_{t}+\varepsilon u \cdot \nabla \xi-\Delta \xi=W^{\prime}\left(W^{\prime}+G^{\prime}\right)-\left|\nabla^{2} \varphi\right|^{2}-\varepsilon \nabla u: \nabla \varphi \otimes \nabla \varphi+G^{\prime \prime}|\nabla \varphi|^{2} .
$$

Differentiating 3.22 with respect to $x_{j}$, we derive, by the Cauchy-Schwarz inequality,

$$
\begin{aligned}
\sum_{j=1}^{d}\left(\sum_{i=1}^{d} \varphi_{x_{i}} \varphi_{x_{i} x_{j}}\right)^{2} & =\sum_{j=1}^{d}\left(\xi_{x_{j}}+\left(W^{\prime}+G^{\prime}\right) \varphi_{x_{j}}\right)^{2} \\
& =|\nabla \xi|^{2}+2\left(W^{\prime}+G^{\prime}\right) \nabla \xi \cdot \nabla \varphi+\left(W^{\prime}+G^{\prime}\right)^{2}|\nabla \varphi|^{2} \leqslant|\nabla \varphi|^{2}\left|\nabla^{2} \varphi\right|^{2} .
\end{aligned}
$$

Divide (3.26) by $|\nabla \varphi|^{2}$ and substitute into (3.25) to obtain

$$
\begin{aligned}
\xi_{t}+\varepsilon u & \nabla \xi-\Delta \xi \\
\leqslant & W^{\prime}\left(W^{\prime}+G^{\prime}\right)-\frac{1}{|\nabla \varphi|^{2}}\left(|\nabla \xi|^{2}+2\left(W^{\prime}+G^{\prime}\right) \nabla \xi \cdot \nabla \varphi+\left(W^{\prime}+G^{\prime}\right)^{2}|\nabla \varphi|^{2}\right) \\
& \quad-\varepsilon \nabla u: \nabla \varphi \otimes \nabla \varphi+G^{\prime \prime}|\nabla \varphi|^{2} \\
\leqslant & -\left(G^{\prime}\right)^{2}-W^{\prime} G^{\prime}-\frac{2\left(W^{\prime}+G^{\prime}\right)}{|\nabla \varphi|^{2}} \nabla \xi \cdot \nabla \varphi-\varepsilon \nabla u: \nabla \varphi \otimes \nabla \varphi+G^{\prime \prime}|\nabla \varphi|^{2} .
\end{aligned}
$$


By substituting $|\nabla \varphi|^{2}=2 \xi+2(W+G)$ into 3.27 ,

$$
\begin{aligned}
\xi_{t}+\varepsilon u \cdot \nabla \xi-\Delta \xi \leqslant & -\left(G^{\prime}\right)^{2}-W^{\prime} G^{\prime}+2 G^{\prime \prime}(W+G)-\frac{2\left(W^{\prime}+G^{\prime}\right)}{|\nabla \varphi|^{2}} \nabla \xi \cdot \nabla \varphi \\
& -\varepsilon \nabla u: \nabla \varphi \otimes \nabla \varphi+2 G^{\prime \prime} \xi
\end{aligned}
$$

Let $\phi(x, t)=\phi(x) \in C^{\infty}\left(B_{3 \varepsilon^{-1}}(0)\right)$ be such that

$$
\phi= \begin{cases}M=\sup _{\mathbb{R}^{d} \times\left[0, \varepsilon^{-2} T\right]}\left(|\nabla \varphi|^{2} / 2-W(\varphi)\right) & \text { on } B_{3 \varepsilon^{-1}}(0) \backslash B_{2 \varepsilon^{-1}}(0), \\ 0 & \text { on } B_{\varepsilon^{-1}}(0),\end{cases}
$$

and

$$
0 \leqslant \phi \leqslant M, \quad|\nabla \phi| \leqslant 2 \varepsilon M, \quad|\Delta \phi| \leqslant 2 d \varepsilon^{2} M
$$

Note that $M$ may be bounded depending only on $d, W, c_{1}, c_{2}$ by Lemma 3.3 . We then set

$$
\tilde{\xi}=\xi-\phi \quad \text { and } \quad G(\varphi)=\varepsilon^{1 / 2}\left(1-\frac{1}{8} \varphi^{2}\right)
$$

Suppose, to derive a contradiction, that

$$
\sup _{B_{\varepsilon^{-1}} \times\left[0, \varepsilon^{-2} T\right]} \xi \geqslant \varepsilon^{1 / 2}
$$

Since $\tilde{\xi} \leqslant 0$ on $\left(B_{3 \varepsilon^{-1}} \backslash B_{\varepsilon^{-1}}\right) \times\left[0, \varepsilon^{-2} T\right]$ and $\tilde{\xi} \leqslant \varepsilon^{1-\gamma}$ on $B_{3 \varepsilon^{-1}} \times\{0\}$ by (3.4), and since $\sup _{B_{\varepsilon^{-1}} \times\left[0, \varepsilon^{-2} \tilde{T}\right]} \tilde{\xi} \geqslant \varepsilon^{1 / 2}$, there exists some interior maximum point $\left(x_{0}, t_{0}\right)$ of $\tilde{\xi}$ where

$$
\tilde{\xi}_{t} \geqslant 0, \quad \nabla \tilde{\xi}=0, \quad \Delta \tilde{\xi} \leqslant 0, \quad \tilde{\xi} \geqslant \varepsilon^{1 / 2} .
$$

With 3.29, we have at this point

$$
\xi_{t} \geqslant 0, \quad \|\left.\nabla \xi\left|\leqslant 2 \varepsilon M, \quad \Delta \xi \leqslant 2 d \varepsilon^{2} M, \quad\right| \nabla \varphi\right|^{2} \geqslant 2 \varepsilon^{1 / 2} .
$$

Substitute (3.30) into 3.28). Using $\varepsilon \nabla u: \nabla \varphi \otimes \nabla \varphi \leqslant 2 \varepsilon|\nabla u|(\xi+W+G)$ and (3.5), we have

$$
\begin{aligned}
0 \leqslant & 2 d \varepsilon^{2} M-\left(G^{\prime}\right)^{2}-W^{\prime} G^{\prime}+2 G^{\prime \prime}(W+G)+\frac{4\left(\left|W^{\prime}\right|+\left|G^{\prime}\right|\right) \varepsilon M}{\left(2 \varepsilon^{1 / 2}\right)^{1 / 2}} \\
& +2 G^{\prime \prime} \xi+2 \varepsilon^{1-\gamma} c_{2}(\xi+W+G)+2 c_{2} \varepsilon^{2-\gamma} M
\end{aligned}
$$

We have $W^{\prime} G^{\prime} \geqslant 0, G^{\prime \prime}=-\varepsilon^{1 / 2} / 4<0$, and since $\gamma<1 / 2$, for sufficiently small $\varepsilon=\varepsilon\left(W, c_{2}, \gamma\right)$,

$$
2 G^{\prime \prime} \xi+2 c_{2} \varepsilon^{1-\gamma} \xi \leqslant 0, \quad 2 G^{\prime \prime}(W+G)+2 c_{2} \varepsilon^{1-\gamma}(W+G) \leqslant G^{\prime \prime}(W+G) .
$$

If $\left|\varphi\left(x_{0}, t_{0}\right)\right| \leqslant 1 / 2$, then

$$
G^{\prime \prime} W \leqslant-\frac{\varepsilon^{1 / 2}}{2} \min _{|z| \leqslant 1 / 2} W(z)
$$


which is a 'big' negative number compared to the rest, and one can check that this and (3.32) lead to a contradiction in 3.31. If $\left|\varphi\left(x_{0}, t_{0}\right)\right| \geqslant 1 / 2$, then we would have 'big' negative contributions coming from

$$
-\left(G^{\prime}\right)^{2} \leqslant-\frac{1}{64} \varepsilon \quad \text { and } \quad-W^{\prime} G^{\prime} \leqslant-\frac{1}{8} \varepsilon^{1 / 2}\left|W^{\prime}\right|,
$$

which again leads to a contradiction with 3.31 for sufficiently small $\varepsilon$. This shows that (since $\left.G \leqslant \varepsilon^{1 / 2}\right)$

$$
\sup _{B_{\varepsilon^{-1}} \times\left[0, \varepsilon^{-2} T\right]}\left(|\nabla \varphi|^{2} / 2-W(\varphi)\right) \leqslant 2 \varepsilon^{1 / 2} .
$$

Now repeat the same argument with $M$ replaced by $2 \varepsilon^{1 / 2}$ and $G$ replaced by $8 c_{2} \varepsilon^{1-\gamma}\left(1-\frac{1}{8} \varphi^{2}\right)$. If we assume

$$
\sup _{B_{\varepsilon^{-1}} \times\left[0, \varepsilon^{-2} T\right]} \xi \geqslant 2 \varepsilon^{1-\gamma},
$$

$\tilde{\xi}=\xi-\phi$ would attain some interior maximum in $B_{3 \varepsilon^{-1}} \times\left[0, \varepsilon^{-2} T\right]$ by (3.4) and by the subtraction of $\phi$. This time we would have $\xi_{t} \leqslant 0,|\nabla \xi| \leqslant 4 \varepsilon^{3 / 2}, \Delta \xi \leqslant 4 d \varepsilon^{5 / 2}$ and $|\nabla \varphi|^{2} \geqslant 4 \varepsilon^{1-\gamma}$. Now (3.31) becomes

$$
\begin{aligned}
0 \leqslant & 4 d \varepsilon^{5 / 2}-\left(G^{\prime}\right)^{2}-W^{\prime} G^{\prime}+2 G^{\prime \prime}(W+G)+\frac{8\left(\left|W^{\prime}\right|+\left|G^{\prime}\right|\right) \varepsilon^{3 / 2}}{\left(4 \varepsilon^{1-\gamma}\right)^{1 / 2}} \\
& +2 G^{\prime \prime} \xi+2 \varepsilon^{1-\gamma} c_{2}(\xi+W+G)+4 \varepsilon^{5 / 2-\gamma} c_{2} .
\end{aligned}
$$

Exactly the same type of argument as before shows that we have a contradiction, and since $G \leqslant$ $8 c_{2} \varepsilon^{1-\gamma}$ and $\xi-G \leqslant 2 \varepsilon^{1-\gamma}$, we obtain 3.21.

Lemma 3.5 For $s, R, r>0$ with $0 \leqslant s-(R / r)^{2} \leqslant T$ and $R \leqslant 1 / 2$, there exists $c_{9}=c_{9}(d) \geqslant 1$ such that

$$
\begin{aligned}
& \int_{\Omega} \tilde{\rho}_{(y, s)}(x, \tilde{s}) \mathrm{d} \mu_{\tilde{s}}(x) \\
& \quad \leqslant(r /(\sqrt{4 \pi} R))^{d-1}\left\{\mu_{\tilde{s}}\left(B_{R}(y)\right)+\mu_{\tilde{s}}(\Omega) \exp \left(-r^{2} /\left(16 R^{2}\right)\right)\right\}+c_{9} D(\tilde{s}) \exp \left(-r^{2} / 8\right) .
\end{aligned}
$$

Here $\tilde{s}=s-(R / r)^{2}$.

Proof. We note that

$$
\tilde{\rho}_{(y, s)}(x, \tilde{s})=\frac{r^{d-1}}{(\sqrt{4 \pi} R)^{d-1}} e^{-\frac{r^{2}|x-y|^{2}}{4 R^{2}}} \eta(x-y) .
$$

We evaluate the integrand separately on $B_{R}(y)$ and on $\Omega \backslash B_{R}(y)$. On $B_{R}(y)$,

$$
\begin{aligned}
\int_{B_{R}(y)} \tilde{\rho}_{(y, s)}(x, \tilde{s}) \mathrm{d} \mu_{\tilde{s}}(x) & \leqslant \int_{B_{R}(y)} \rho_{(y, s)}(x, \tilde{s}) \mathrm{d} \mu_{\tilde{s}}(x)=\frac{r^{d-1}}{(\sqrt{4 \pi} R)^{d-1}} \int_{B_{R}(y)} e^{-\frac{r^{2}|x-y|^{2}}{4 R^{2}}} \mathrm{~d} \mu_{\tilde{s}} \\
& \leqslant \frac{r^{d-1}}{(\sqrt{4 \pi} R)^{d-1}} \mu_{\tilde{s}}\left(B_{R}(y)\right)
\end{aligned}
$$


On $\Omega \backslash B_{R}(y)$ and $R \leqslant 1 / 2$, we use (3.7) to derive

$$
\begin{aligned}
& \frac{(\sqrt{4 \pi} R)^{d-1}}{r^{d-1}} \int_{\Omega \backslash B_{R}(y)} \tilde{\rho}_{(y, s)}(x, \tilde{s}) \mathrm{d} \mu_{\tilde{s}}(x) \leqslant \int_{B_{1 / 2}(y) \backslash B_{R}(y)} e^{-\frac{r^{2}|x-y|^{2}}{4 R^{2}}} \mathrm{~d} \mu_{\tilde{s}}(x) \\
& \quad=\int_{0}^{1} \mu_{\tilde{s}}\left(\left(B_{1 / 2}(y) \backslash B_{R}(y)\right) \cap\left\{x ; e^{-\frac{r^{2}|x-y|^{2}}{4 R^{2}}} \geqslant \lambda\right\}\right) \mathrm{d} \lambda \\
& \quad \leqslant \int_{0}^{\exp \left(-r^{2} /\left(16 R^{2}\right)\right)} \mu_{\tilde{s}}\left(B_{1 / 2}(y) \backslash B_{R}(y)\right) \mathrm{d} \lambda+\int_{\exp \left(-r^{2} /\left(16 R^{2}\right)\right)}^{\exp \left(-r^{2} / 4\right)} \mu_{\tilde{s}}\left(B_{\left.2 R r^{-1} \sqrt{\ln \lambda^{-1}}(y)\right) \mathrm{d} \lambda}\right. \\
& \quad \leqslant E \exp \left(-r^{2} /\left(16 R^{2}\right)\right)+D(\tilde{s}) \omega_{d-1}(2 R / r)^{d-1} \int_{r^{2} / 4}^{r^{2} /\left(16 R^{2}\right)} s^{(d-1) / 2} \exp (-s) \mathrm{d} s \\
& \quad \leqslant E \exp \left(-r^{2} /\left(16 R^{2}\right)\right)+c(d) D(\tilde{s})(R / r)^{d-1} \exp \left(-r^{2} / 8\right) .
\end{aligned}
$$

Here we wrote $E=\mu_{\tilde{s}}(\Omega)$. Combining (3.34) and 3.35 , we obtain 3.33 . If necessary we may choose $c_{9} \geqslant 1$.

\subsection{Proof of Theorem 3.1}

In the following we show that the growth of the density upper ratio is controlled in time in the following sense.

Proposition 3.6 There exist $c_{b}=c_{b}(d)>1, T_{b}=T_{b}\left(d, p, c_{3}\right)>0$ and $\epsilon_{b}=$ $\epsilon_{b}\left(d, p, W, c_{1}, c_{2}, c_{3}, D_{0}, T, \gamma\right)$ such that

$$
\sup _{t \in\left[t_{0}, t_{0}+T_{b}\right]} D(t) \leqslant c_{b} D\left(t_{0}\right)
$$

for all $t_{0} \in\left[0, T-T_{b}\right]$ and $\varepsilon<\epsilon_{b}$.

If this holds, $D(t)$ satisfies

$$
D(t) \leqslant D_{0} c_{b}^{\left[t / T_{b}\right]+1}
$$

where $[x]$ is the integral part of $x$. Thus 3.36 finishes the proof of Theorem 3.1 We assume in the following that $D(t)$ satisfies

$$
D(t) \leqslant D_{0} c_{b}^{\left[T / T_{b}\right]+1}\left(=c_{10}\left(d, p, c_{3}, T, D_{0}\right)\right)
$$

for $t \in\left[0, T_{1}\right]$. Note that $T_{1}>0$ (a priori depending on $\varepsilon$ ) since $c_{b}>1$ and by the continuity of $D(t)$ in time. Under this assumption we have

LEMMA 3.7

$$
\sup _{t \in\left[0, T_{1}\right]} \mu_{t}(\Omega)+\int_{0}^{T_{1}} \int_{\Omega} \frac{\varepsilon}{\sigma}\left(\Delta \varphi-\frac{W^{\prime}(\varphi)}{\varepsilon^{2}}\right)^{2} \mathrm{~d} x \mathrm{~d} t \leqslant E_{0}=E_{0}\left(d, p, c_{3}, T, D_{0}\right) .
$$


264

C. DIU, N. SATI AND Y. TONEGAWA

Proof. By [3.1] we can compute

$$
\frac{\mathrm{d}}{\mathrm{d} t} \mu_{t}(\Omega) \leqslant-\frac{1}{2 \sigma} \int_{\Omega} \varepsilon\left(\Delta \varphi-\frac{W^{\prime}(\varphi)}{\varepsilon^{2}}\right)^{2} \mathrm{~d} x+\frac{\varepsilon}{\sigma} \int_{\Omega}(u \cdot \nabla \varphi)^{2} \mathrm{~d} x .
$$

By (2.4) we have

$$
\frac{\mathrm{d}}{\mathrm{d} t} \mu_{t}(\Omega) \leqslant-\frac{1}{2 \sigma} \int_{\Omega} \varepsilon\left(\Delta \varphi-\frac{W^{\prime}(\varphi)}{\varepsilon^{2}}\right)^{2} \mathrm{~d} x+\int_{\Omega} c_{10} c_{M Z}\left(|u|^{2}+|\nabla u|^{2}\right) \mathrm{d} x .
$$

Thus by integrating with respect to $t$ and by (3.6), we obtain $(3.38)$. Note that $\mu_{0}(\Omega) \leqslant c(d) D_{0}$.

The next Lemma 3.8 gives a lower bound of the density of $\mu_{t}$ when there is some piece of interface. For the rest of the present section we fix $\gamma^{\prime}$ such that $\gamma<\gamma^{\prime}<1 / 2$ and $\gamma^{\prime}=\frac{1}{2}(1 / 2+\gamma), \alpha=3 / 4$ and $\kappa=11 / 6$ so that $W^{\prime \prime}(s) \geqslant \kappa$ for $\alpha \leqslant|s| \leqslant 1$.

LEMMA 3.8 There exist positive constants $c_{11}$ and $c_{12}$ and $\epsilon_{3}$ with $0<\epsilon_{3} \leqslant \epsilon_{2}$ depending only on $d, W, c_{1}, c_{2}, c_{3}, p, D_{0}, \gamma$ and $\gamma^{\prime}$ with the following properties: Suppose $0<\varepsilon<\epsilon_{3}$ and $|\varphi(y, s)| \leqslant \alpha<1$ with $s \in\left[0, T_{1}\right]$. Then, for all $t \in\left[0, T_{1}\right]$ with $\max \left\{0, s-\varepsilon^{2 \gamma^{\prime}}\right\} \leqslant t \leqslant s$ and $\varepsilon c_{11} \leqslant R \leqslant \varepsilon^{\gamma^{\prime}} c_{11}$, we have

$$
c_{12} \leqslant \frac{1}{R^{d-1}} \mu_{t}\left(B_{R}(y)\right) .
$$

Proof. In this proof we write $\tilde{\rho}=\tilde{\rho}_{\left(y, s+\varepsilon^{2}\right)}(x, t)$ for brevity. Suppose $|\varphi(y, s)| \leqslant \alpha<1$. If we change the variable $\tilde{\varphi}(\tilde{x}, s)=\varphi(\varepsilon \tilde{x}+y, s)$, we obtain

$$
\int_{\Omega} \tilde{\rho} \mathrm{d} \mu_{S}(x)=\int_{\Omega / \varepsilon} \frac{e^{-|\tilde{x}|^{2} / 4}}{(\sqrt{4 \pi})^{d-1} \sigma} \eta(\varepsilon \tilde{x})\left(\frac{|\nabla \tilde{\varphi}|^{2}}{2}+W(\tilde{\varphi})\right) \mathrm{d} \tilde{x} .
$$

Since $|\tilde{\varphi}(0, s)| \leqslant \alpha<1$, Lemma 3.3 implies that there exists a positive constant $c_{13}=$ $c_{13}\left(d, W, \gamma, c_{1}, c_{2}\right) \leqslant 1$ such that

$$
5 c_{13} \leqslant \int_{\Omega} \tilde{\rho} \mathrm{d} \mu_{s}(x)
$$

Next, we use 3.5, 3.9] and 3.21 to obtain, for $\lambda \in[t, s)$,

$$
\begin{aligned}
\frac{\mathrm{d}}{\mathrm{d} \lambda} \int_{\Omega} \tilde{\rho} \mathrm{d} \mu_{\lambda} \leqslant & c_{2}^{2} \varepsilon^{-2 \gamma} \int_{\Omega} \tilde{\rho} \mathrm{d} \mu_{\lambda}+\frac{\left(2+8 c_{2}\right) \sqrt{\pi} \varepsilon^{-\gamma}}{\sigma \sqrt{s-\lambda}} \\
& +c_{5} e^{\frac{-c_{6}}{s+\varepsilon^{2}-\lambda}}\left(E_{0}+\int_{\Omega} \varepsilon\left(\Delta \varphi-\frac{W^{\prime}}{\varepsilon^{2}}\right)^{2} \mathrm{~d} x\right) .
\end{aligned}
$$

Multiply (3.41) by $e^{c_{2}^{2} \varepsilon^{-2 \gamma}(s-\lambda)}$ and integrate over $[t, s]$. Here we use $t \geqslant s-\varepsilon^{2 \gamma^{\prime}}$ and 3.38) to estimate the right-hand side as

$$
\begin{aligned}
e^{c_{2}^{2} \varepsilon^{-2 \gamma}(s-\lambda)} & \left.\int_{\Omega} \tilde{\rho} \mathrm{d} \mu_{\lambda}(x)\right|_{\lambda=t} ^{s} \\
\leqslant & \varepsilon^{\gamma^{\prime}-\gamma} e^{c_{2}^{2} \varepsilon^{2\left(\gamma^{\prime}-\gamma\right)}} \sigma^{-1} 2\left(2+8 c_{2}\right) \sqrt{\pi}+c_{5} E_{0} e^{c_{2}^{2} \varepsilon^{2\left(\gamma^{\prime}-\gamma\right)}} e^{-\frac{c_{6}}{\varepsilon^{2}+\varepsilon^{2 \gamma^{\prime}}}}\left(1+\varepsilon^{2 \gamma^{\prime}}\right) .
\end{aligned}
$$


Next, we restrict $\varepsilon$ to be so small that the right-hand side of $(3.42)$ is less than $c_{13}$ and $e^{c_{2}^{2} \varepsilon^{2\left(\gamma^{\prime}-\gamma\right)}} \leqslant 2$. Then, for $t \in\left[0, T_{1}\right]$ with $s-\varepsilon^{2 \gamma^{\prime}}<t<s$, (3.40) and (3.42) show

$$
2 c_{13} \leqslant \int_{\Omega} \tilde{\rho} \mathrm{d} \mu_{t}(x)
$$

Next we use Lemma 3.5 with $r=\sqrt{8 \ln \left(2 c_{9} c_{10} / c_{13}\right)}$, where the argument of $\ln$ is $\geqslant 2$ due to $c_{9} \geqslant 1, c_{10} \geqslant 1$ and $c_{13} \leqslant 1$. The equality yields

$$
c_{9} c_{10} e^{-r^{2} / 8}=c_{13} / 2 .
$$

In Lemma 3.5. we replace $s$ and $s-(R / r)^{2}$ by $s+\varepsilon^{2}$ and $t$ respectively, so that $R^{2}=r^{2}\left(s+\varepsilon^{2}-t\right)$. Note that since $s-\varepsilon^{2 \gamma^{\prime}} \leqslant t<s, R \leqslant r \sqrt{\varepsilon^{2}+\varepsilon^{2 \gamma^{\prime}}}$ and we have $R \leqslant 1 / 2$ by restricting $\varepsilon$ depending only on $c_{9}, c_{10}, c_{13}$. From Lemma 3.5 we obtain

$$
\int_{\Omega} \tilde{\rho} \mathrm{d} \mu_{t}(x) \leqslant(r /(\sqrt{4 \pi} R))^{d-1}\left\{\mu_{t}\left(B_{R}(y)\right)+E_{0} e^{-r^{2} /\left(16 R^{2}\right)}\right\}+c_{9} c_{10} e^{-r^{2} / 8} .
$$

Due to 3.44, (3.43) and restricting $\varepsilon$ further we obtain

$$
c_{13} \leqslant(r /(\sqrt{4 \pi} R))^{d-1} \mu_{t}\left(B_{R}(y)\right) .
$$

Note that $R$ lies in $\left[r \varepsilon, r \sqrt{\left(\varepsilon^{2}+\varepsilon^{2 \gamma^{\prime}}\right)}\right]$, so setting $c_{11}=r$ and $c_{12}=r^{1-d}(\sqrt{4 \pi})^{d-1} c_{13}$ we have the desired estimate.

Lemma 3.9 There exist $\epsilon_{4} \leqslant \epsilon_{3}$ and $c_{14}$ depending only on $d, W, c_{1}, c_{2}, c_{3}, p, D_{0}$ and $\gamma$ with the following property. For $1 / 3 \geqslant r \geqslant \varepsilon^{\gamma^{\prime}}$ and $\varepsilon \leqslant \epsilon_{4}$,

$$
\frac{1}{\sigma} \int_{B_{r}(y)}\left(\frac{\varepsilon|\nabla \varphi|^{2}}{2}-\frac{W(\varphi)}{\varepsilon}\right)_{+}(x, t) \mathrm{d} x \leqslant c_{14} \varepsilon^{\gamma^{\prime}} r^{d-1}
$$

for $t \in\left[\varepsilon^{2 \gamma^{\prime}}, T\right] \cap\left[0, T_{1}\right]$.

Proof. If $T_{1}<\varepsilon^{2 \gamma^{\prime}}$ there is nothing to prove. Assume that $T_{1} \geqslant \varepsilon^{2 \gamma^{\prime}}$ and $t_{*} \in\left[\varepsilon^{2 \gamma^{\prime}}, T\right] \cap\left[0, T_{1}\right]$. In the following, we estimate the integrand on two sets, near the interface and on the complement. Define

$$
\begin{aligned}
& \tilde{A}=\left\{x \in B_{2 r}(y) \mid \text { for some } \tilde{t} \text { with } t_{*}-\varepsilon^{2 \gamma^{\prime}} \leqslant \tilde{t} \leqslant t_{*},|\varphi(x, \tilde{t})| \leqslant \alpha\right\}, \\
& A=\left\{x \in B_{2 r+\varepsilon^{2 \gamma^{\prime}}}(y) \mid \operatorname{dist}(\tilde{A}, x)<\varepsilon^{2 \gamma^{\prime}}\right\} .
\end{aligned}
$$

Points belonging to $\tilde{A}$ have some piece of interface during $\left[t_{*}-\varepsilon^{2 \gamma^{\prime}}, t_{*}\right]$, and $A$ is the $\varepsilon^{2 \gamma^{\prime}}$ neighborhood of $\tilde{A}$. By Vitali's covering theorem [10, 1.5.1] applied to $\mathcal{F}=\left\{\bar{B}_{\varepsilon^{2 \gamma^{\prime}}}(x) \mid x \in \tilde{A}\right\}$ (note $A \subset \bigcup_{B \in \mathcal{F}} B$ ), we can choose a set $\left\{B_{\varepsilon^{2} \gamma^{\prime}}\left(x_{i}\right)\right\}_{i=1}^{N}$ of pairwise disjoint balls such that

$$
x_{i} \in \tilde{A} \quad \text { for each } i=1, \ldots, N \quad \text { and } \quad A \subset \bigcup_{i=1}^{N} \bar{B}_{5 \varepsilon^{2 \gamma^{\prime}}}\left(x_{i}\right) .
$$


For each $x_{i}$, let $\tilde{t}_{i}$ be such that

$$
t_{*}-\varepsilon^{2 \gamma^{\prime}} \leqslant \tilde{t}_{i} \leqslant t_{*}, \quad\left|\varphi\left(x_{i}, \tilde{t}_{i}\right)\right| \leqslant \alpha .
$$

Write $\hat{t}=t_{*}-\varepsilon^{2 \gamma^{\prime}}$. We next use Lemma 3.8 By (3.49), $\tilde{t}_{i}-\hat{t} \leqslant t_{*}-\left(t_{*}-\varepsilon^{2 \gamma^{\prime}}\right)=\varepsilon^{2 \gamma^{\prime}}$ and the assumption of Lemma 3.8 is satisfied for $s=\tilde{t}_{i}, y=x_{i}, t=\hat{t}$ and $R=\varepsilon^{2 \gamma^{\prime}}$ where we restrict $\varepsilon$ depending on $c_{11}$ and $\gamma^{\prime}$. Thus, we conclude

$$
c_{12} \varepsilon^{2 \gamma^{\prime}(d-1)} \leqslant \mu_{\hat{t}}\left(B_{\varepsilon^{2 \gamma^{\prime}}}\left(x_{i}\right)\right) \text { for } i=1, \ldots, N .
$$

Since $\left\{B_{\varepsilon^{2 \gamma^{\prime}}}\left(x_{i}\right)\right\}_{i=1}^{N}$ are pairwise disjoint and $B_{\varepsilon^{2 \gamma^{\prime}}}\left(x_{i}\right) \subset B_{2 r+\varepsilon^{2 \gamma^{\prime}}}(y), 3.50$ gives

$$
N c_{12} \varepsilon^{2 \gamma^{\prime}(d-1)} \leqslant \mu_{\hat{t}}\left(B_{2 r+\varepsilon^{2 \gamma^{\prime}}}(y)\right) .
$$

Then the $d$-dimensional volume of $A$ is estimated using 3.48 and 3.51 as

$$
\mathcal{L}^{d}(A) \leqslant N \omega_{d}\left(5 \varepsilon^{2 \gamma^{\prime}}\right)^{d} \leqslant \frac{\omega_{d} 5^{d} \varepsilon^{2 \gamma^{\prime}}}{c_{12}} \mu_{\hat{t}}\left(B_{2 r+\varepsilon^{2 \gamma^{\prime}}}(y)\right) .
$$

Since $\hat{t} \in\left[0, T_{1}\right]$, by 3.7, 3.37 and 3.52 with $r \geqslant \varepsilon^{2 \gamma^{\prime}}$,

$$
\mathcal{L}^{d}(A) \leqslant \frac{\omega_{d} 5^{d} \varepsilon^{2 \gamma^{\prime}}}{c_{12}} c_{10} \omega_{d-1}\left(2 r+\varepsilon^{2 \gamma^{\prime}}\right)^{d-1} \leqslant c_{15} \varepsilon^{2 \gamma^{\prime}} r^{d-1}
$$

where $c_{15}=\omega_{d} \omega_{d-1} 5^{d} 3^{d-1} c_{10} / c_{12}$. Thus by 3.21, 3.53 and $\gamma^{\prime}>\gamma$,

$$
\int_{A \cap B_{r}(y)}\left(\frac{\varepsilon|\nabla \varphi|^{2}}{2}-\frac{W(\varphi)}{\varepsilon}\right)_{+}\left(x, t_{*}\right) \mathrm{d} x \leqslant \mathcal{L}^{d}(A)\left(2+8 c_{2}\right) \varepsilon^{-\gamma} \leqslant\left(2+8 c_{2}\right) c_{15} \varepsilon^{\gamma^{\prime}} r^{d-1}
$$

Next we show that the surface energy decays quickly on the complement of $A$. Fix the integer $J \geqslant 2$ such that

$$
(J-1)\left(2-4 \gamma^{\prime}\right) \geqslant \gamma^{\prime}, \quad(J-2)\left(2-4 \gamma^{\prime}\right)<\gamma^{\prime} .
$$

Since $\gamma^{\prime}<1 / 2$, such a $J$ does exist. Define

$$
t_{j}=t_{*}-\varepsilon^{2 \gamma^{\prime}}+\frac{j}{J} \varepsilon^{2 \gamma^{\prime}} \quad(j=0,1, \ldots, J) .
$$

Next define $\phi_{j} \in \operatorname{Lip}\left(B_{2 r}(y)\right)(j=1, \ldots, J)$ so that

$$
\begin{gathered}
\phi_{j}(x)=\phi_{j}(x, t)= \begin{cases}1 & \text { if } \operatorname{dist}\left(x, B_{r}(y) \backslash A\right) \leqslant \frac{J-j}{J} \varepsilon^{2 \gamma^{\prime}}, \\
0 & \text { if } \operatorname{dist}\left(x, B_{r}(y) \backslash A\right) \geqslant \frac{J-j+1}{J} \varepsilon^{2 \gamma^{\prime}},\end{cases} \\
\left|\nabla \phi_{j}\right| \leqslant 2 J \varepsilon^{-2 \gamma^{\prime}} \text { and } 0 \leqslant \phi_{j} \leqslant 1 .
\end{gathered}
$$

By (3.56, 3.46), 3.47) and $r \geqslant \varepsilon^{\gamma^{\prime}}$, one can check that $\operatorname{spt} \phi_{j} \cap \tilde{A}=\emptyset$ for $j=1, \ldots, J$, thus by (3.46,

$$
|\varphi(x, s)| \geqslant \alpha \quad \text { for } x \in \operatorname{spt} \phi_{j}, s \in\left[t_{*}-\varepsilon^{2 \gamma^{\prime}}, t_{*}\right] .
$$


For each $j=1, \ldots, J$, differentiate the equation (3.1) with respect to $x_{i}$, multiply by $\phi_{j}^{2} \partial \varphi / \partial x_{i}$, sum over $i$ and integrate to obtain

$$
\begin{aligned}
\frac{\mathrm{d}}{\mathrm{d} t} \int_{\Omega} \frac{1}{2}|\nabla \varphi|^{2} \phi_{j}^{2} \mathrm{~d} x+\int_{\Omega}\left(u \otimes \nabla \varphi: \nabla^{2} \varphi\right. & +\nabla \varphi \otimes \nabla \varphi: \nabla u) \phi_{j}^{2} \mathrm{~d} x \\
& =\int_{\Omega}\left(\nabla \varphi \cdot \Delta \nabla \varphi-\frac{W^{\prime \prime}(\varphi)}{\varepsilon^{2}}|\nabla \varphi|^{2}\right) \phi_{j}^{2} \mathrm{~d} x .
\end{aligned}
$$

By integration by parts and the Cauchy-Schwarz inequality we obtain from 3.59]

$$
\begin{aligned}
\frac{\mathrm{d}}{\mathrm{d} t} \int_{\Omega} \frac{1}{2}|\nabla \varphi|^{2} \phi_{j}^{2} \mathrm{~d} x \leqslant & \frac{1}{2} \int_{\Omega}|u|^{2}|\nabla \varphi|^{2} \phi_{j}^{2} \mathrm{~d} x+\int_{\Omega}|\nabla \varphi|^{2}|\nabla u| \phi_{j}^{2} \mathrm{~d} x \\
& +4 \int_{\Omega}\left|\nabla \phi_{j}\right|^{2}|\nabla \varphi|^{2} \mathrm{~d} x-\int_{\Omega} \frac{W^{\prime \prime}(\varphi)}{\varepsilon^{2}}|\nabla \varphi|^{2} \phi_{j}^{2} \mathrm{~d} x .
\end{aligned}
$$

By [3.58), $W^{\prime \prime}(\varphi) \geqslant \kappa$ on spt $\phi_{j}$ for $t \in\left[t_{*}-\varepsilon^{2 \gamma^{\prime}}, t_{*}\right]$. Using also 3.57) and [3.5, we obtain

$$
\begin{aligned}
& \frac{\mathrm{d}}{\mathrm{d} t} \int_{\Omega} \frac{1}{2}|\nabla \varphi|^{2} \phi_{j}^{2} \mathrm{~d} x \\
& \quad \leqslant \int_{\Omega}\left(\frac{\varepsilon^{-2 \gamma}}{2}+\varepsilon^{-1-\gamma}\right)|\nabla \varphi|^{2} \phi_{j}^{2} \mathrm{~d} x+16 J^{2} \varepsilon^{-4 \gamma^{\prime}} \int_{\operatorname{spt} \phi_{j}}|\nabla \varphi|^{2} \mathrm{~d} x-\frac{\kappa}{\varepsilon^{2}} \int_{\Omega}|\nabla \varphi|^{2} \phi_{j}^{2} \mathrm{~d} x \\
& \quad \leqslant-\frac{\kappa}{2 \varepsilon^{2}} \int_{\Omega}|\nabla \varphi|^{2} \phi_{j}^{2} \mathrm{~d} x+16 J^{2} \varepsilon^{-4 \gamma^{\prime}} \int_{\text {spt } \phi_{j}}|\nabla \varphi|^{2} \mathrm{~d} x
\end{aligned}
$$

for small $\varepsilon$. By integrating (3.61) over $\left[t_{j-1}, s\right]$ with $s \in\left[t_{j}, t_{*}\right], j=1, \ldots, J$, we obtain

$$
\begin{aligned}
\int_{\Omega} \frac{1}{2}|\nabla \varphi|^{2} \phi_{j}^{2}(x, s) \mathrm{d} x \leqslant & e^{-\frac{\kappa}{\varepsilon^{2}}\left(s-t_{j-1}\right)} \int_{\Omega} \frac{1}{2}|\nabla \varphi|^{2} \phi_{j}^{2}\left(x, t_{j-1}\right) \mathrm{d} x \\
& +\int_{t_{j-1}}^{s} e^{-\frac{\kappa}{\varepsilon^{2}}(s-\lambda)} 16 J^{2} \varepsilon^{-4 \gamma^{\prime}}\left(\int_{\operatorname{spt} \phi_{j}}|\nabla \varphi|^{2}(x, \lambda) \mathrm{d} x\right) \mathrm{d} \lambda .
\end{aligned}
$$

For $j=1, \ldots, J$ define

$$
M_{j}=\sup _{\lambda \in\left[t_{j-1}, t_{*}\right]} \int_{\operatorname{spt} \phi_{j}} \frac{1}{2}|\nabla \varphi|^{2}(x, \lambda) \mathrm{d} x
$$

Since $s-t_{j-1} \geqslant t_{j}-t_{j-1}=\frac{1}{J} \varepsilon^{2 \gamma^{\prime}}$ for $s \in\left[t_{j}, t_{*}\right]$, 3.62 with the notation of 3.63) shows

$$
\begin{aligned}
\int_{\Omega} \frac{1}{2}|\nabla \varphi|^{2} \phi_{j}^{2}(x, s) \mathrm{d} x & \leqslant\left(e^{-\frac{\kappa}{\varepsilon^{2}}\left(s-t_{j-1}\right)}+16 \kappa^{-1} J^{2} \varepsilon^{2-4 \gamma^{\prime}}\right) M_{j} \\
& \leqslant\left(e^{-\frac{\kappa}{J \varepsilon^{2-2 \gamma^{\prime}}}}+16 \kappa^{-1} J^{2} \varepsilon^{2-4 \gamma^{\prime}}\right) M_{j} .
\end{aligned}
$$

By (3.56), spt $\phi_{j+1} \subset\left\{\phi_{j}=1\right\}$. Thus by 3.64) and for $j=1, \ldots, J-1$ we have

$$
M_{j+1} \leqslant\left(e^{-\frac{\kappa}{J \varepsilon^{2-2 \gamma^{\prime}}}}+16 \kappa^{-1} J^{2} \varepsilon^{2-4 \gamma^{\prime}}\right) M_{j}
$$


With a suitable restriction on $\varepsilon$ depending only on $W$ and $\gamma^{\prime}\left(<\frac{1}{2}\right), 3.65$ for $j=1, \ldots, J-1$ gives

$$
M_{j+1} \leqslant 17 \kappa^{-1} J^{2} \varepsilon^{2-4 \gamma^{\prime}} M_{j}
$$

Thus, by 3.66) and 3.55,

$$
M_{J} \leqslant\left(17 \kappa^{-1} J^{2}\right)^{J-1} \varepsilon^{\left(2-4 \gamma^{\prime}\right)(J-1)} M_{1} \leqslant\left(17 \kappa^{-1} J^{2}\right)^{J-1} \varepsilon^{\gamma^{\prime}} M_{1} .
$$

From spt $\phi_{1} \subset B_{2 r}(y)$ and 3.37 ,

$$
\varepsilon M_{1}=\sup _{\lambda \in\left[t_{0}, t_{*}\right]} \int_{\operatorname{spt} \phi_{1}} \frac{\varepsilon}{2}|\nabla \varphi|^{2}(x, \lambda) \mathrm{d} x \leqslant \omega_{d-1} c_{10}(2 r)^{d-1} .
$$

Since $B_{r}(y) \backslash A \subset\left\{\phi_{J}=1\right\}$ by 3.56 , we have

$$
\int_{B_{r}(y) \backslash A} \frac{\varepsilon}{2}|\nabla \varphi|^{2}\left(x, t_{*}\right) \mathrm{d} x \leqslant \int_{\left\{\phi_{J}=1\right\}} \frac{\varepsilon}{2}|\nabla \varphi|^{2}\left(x, t_{*}\right) \phi_{J}^{2} \mathrm{~d} x \leqslant \varepsilon M_{J} .
$$

Thus combining 3.67, 3.68 and 3.69) we obtain

$$
\int_{B_{r}(y) \backslash A} \frac{\varepsilon}{2}|\nabla \varphi|^{2}\left(x, t_{*}\right) \mathrm{d} x \leqslant\left(17 \kappa^{-1} J^{2}\right)^{J-1} \varepsilon^{\gamma^{\prime}} c_{10} \omega_{d-1}(2 r)^{d-1} .
$$

With a suitable choice of $c_{14}$ in 3.54) and 3.70 we obtain 3.45).

LEMmA 3.10 There exists a constant $c_{16}$ such that for $\varepsilon \leqslant \epsilon_{4}, t \in\left[0, T_{1}\right]$ and $t<s$, we have

$$
\int_{0}^{t}\left\{\frac{1}{2(s-\lambda)} \int_{\Omega}\left(\frac{\varepsilon|\nabla \varphi|^{2}}{2}-\frac{W(\varphi)}{\varepsilon}\right)_{+} \tilde{\rho}_{(y, s)}(x, \lambda) \mathrm{d} x\right\} \mathrm{d} \lambda \leqslant c_{16} \varepsilon^{\gamma^{\prime}-\gamma} .
$$

Proof. In case $t \leqslant \varepsilon^{2 \gamma^{\prime}}$, using 3.21 and $\int_{\mathbb{R}^{d}} \rho_{(y, s)}(x, \lambda) \mathrm{d} x=\sqrt{4 \pi(s-\lambda)}$, we have

$$
\begin{aligned}
\int_{0}^{t} \frac{1}{2(s-\lambda)} \int_{\Omega}\left(\frac{\varepsilon|\nabla \varphi|^{2}}{2}-\frac{W(\varphi)}{\varepsilon}\right)_{+} \tilde{\rho}_{(y, s)}(x, \lambda) \mathrm{d} x \mathrm{~d} \lambda & \leqslant \int_{0}^{t} \frac{\left(2+8 c_{2}\right) \varepsilon^{-\gamma} \sqrt{\pi}}{\sqrt{s-\lambda}} \mathrm{d} \lambda \\
& \leqslant\left(2+8 c_{2}\right) \sqrt{\pi} \varepsilon^{\gamma^{\prime}-\gamma}
\end{aligned}
$$

In case $s>t \geqslant s-\varepsilon^{2 \gamma^{\prime}}$, we have similarly

$$
\int_{s-\varepsilon^{2 \gamma^{\prime}}}^{t} \frac{1}{2(s-\lambda)} \int_{\Omega}\left(\frac{\varepsilon|\nabla \varphi|^{2}}{2}-\frac{W(\varphi)}{\varepsilon}\right)_{+} \tilde{\rho} \mathrm{d} x \mathrm{~d} \lambda \leqslant\left(2+8 c_{2}\right) \sqrt{\pi} \varepsilon^{\gamma^{\prime}-\gamma} .
$$

Thus we only need to estimate the integral over $\left[\varepsilon^{2 \gamma^{\prime}}, t\right]$ with $t \leqslant s-\varepsilon^{2 \gamma^{\prime}}$. When integrating over $\Omega$, we integrate on $B_{\varepsilon \gamma^{\prime}}(y)$ and $\Omega \backslash B_{\varepsilon} \gamma^{\prime}(y)$ separately. For the first estimate we use 3.21) and $s-t \geqslant \varepsilon^{2 \gamma^{\prime}}$ to estimate

$$
\begin{aligned}
\int_{\varepsilon^{2 \gamma^{\prime}}}^{t} \frac{1}{2(s-\lambda)} \int_{B_{\varepsilon \gamma^{\prime}}}\left(\frac{\varepsilon|\nabla \varphi|^{2}}{2}-\frac{W(\varphi)}{\varepsilon}\right)_{+} \tilde{\rho} \mathrm{d} x \mathrm{~d} \lambda & \leqslant \int_{\varepsilon^{2 \gamma^{\prime}}}^{t} \frac{\left(2+8 c_{2}\right) \varepsilon^{-\gamma} \varepsilon^{d \gamma^{\prime}} \omega_{d}}{(\sqrt{4 \pi})^{d-1} 2(s-\lambda)^{(d+1) / 2} \mathrm{~d} \lambda} \\
& \leqslant \frac{\left(2+8 c_{2}\right) \varepsilon^{\gamma^{\prime}-\gamma} \omega_{d}}{(\sqrt{4 \pi})^{d-1}(d-1)}
\end{aligned}
$$


On $\Omega \backslash B_{\varepsilon \gamma^{\prime}}(y)$, by Lemma 3.9 and $s-t \geqslant \varepsilon^{2 \gamma^{\prime}}$, computations similar to 3.35) give

$\int_{\varepsilon^{2} \gamma^{\prime}}^{t} \frac{1}{2(s-\lambda)} \int_{\Omega \backslash B_{\varepsilon \gamma^{\prime}}(y)}\left(\frac{\varepsilon|\nabla \varphi|^{2}}{2}-\frac{W(\varphi)}{\varepsilon}\right)_{+} \tilde{\rho} \mathrm{d} x \mathrm{~d} \lambda$

$\leqslant \int_{\varepsilon^{2} \gamma^{\prime}}^{t} \frac{\mathrm{d} \lambda}{2(\sqrt{4 \pi})^{(d-1) / 2}(s-\lambda)^{(d+1) / 2}} \int_{0}^{1}\left\{\int_{B_{1 / 2}(y) \cap\left\{x \mid e^{-\frac{|x-y|^{2}}{4(s-\lambda)}} \geqslant q\right\} \backslash B_{\varepsilon} \gamma^{\prime}(y)}\left(\frac{\varepsilon|\nabla \varphi|^{2}}{2}-\frac{W(\varphi)}{\varepsilon}\right)_{+}\right\} \mathrm{d} q$

$\leqslant \int_{\varepsilon^{2} \gamma^{\prime}}^{t} \frac{c_{14} c(d) \varepsilon^{\gamma^{\prime}}\left(e^{-1 /(16(s-\lambda))}+(s-\lambda)^{d-1 / 2}\right)}{(s-\lambda)^{(d+1) / 2}} \mathrm{~d} \lambda \leqslant c_{14} \varepsilon^{\gamma^{\prime}}\left(c(d)+\gamma^{\prime} \ln (1 / \varepsilon)\right)$.

Combining (3.72)-3.75) we obtain the desired estimate 3.71).

Next we estimate the effect of velocity terms in (3.9). Since $\Omega$ is bounded, the $p=d$ case is covered by choosing $p^{\prime}<d$. The argument for $p>d$ is similar and simpler than for $p<d$ so we omit the proof.

LEmma 3.11 Suppose $(d+2) / 2<p<d$. Then there exists a constant $c_{18}=c_{18}(d, p)$ such that for all $s>t_{1}>t_{0} \geqslant 0$,

$$
\int_{t_{0}}^{t_{1}} \int_{\Omega} \tilde{\rho}_{(y, s)}|u|^{2} \mathrm{~d} \mu_{t} \mathrm{~d} t \leqslant c_{18}\left(t_{1}-t_{0}\right)^{\frac{2 p-d-2}{p-2}}\left(\sup _{t \in\left[t_{0}, t_{1}\right]} D(t)\right)\left(\int_{t_{0}}^{t_{1}}\|u(\cdot, t)\|_{W^{1, p}(\Omega)}^{p} \mathrm{~d} t\right)^{2 / p} .
$$

For $p>d$ we have

$$
\int_{t_{0}}^{t_{1}} \int_{\Omega} \tilde{\rho}_{(y, s)}|u|^{2} \mathrm{~d} \mu_{t} \mathrm{~d} t \leqslant\left(\sup _{t \in\left[t_{0}, t_{1}\right]} D(t)\right) \int_{t_{0}}^{t_{1}}\|u(\cdot, t)\|_{L^{\infty}(\Omega)}^{2} \mathrm{~d} t .
$$

Proof. By the Hölder inequality and 3.37,

$$
\begin{aligned}
\int_{\Omega} \tilde{\rho}|u|^{2} \mathrm{~d} \mu_{t} & \leqslant\left(\int_{\Omega}|u|^{2 l} \tilde{\rho} \mathrm{d} \mu_{t}\right)^{1 / l}\left(\int_{\Omega} \tilde{\rho} \mathrm{d} \mu_{t}\right)^{(l-1) / l} \leqslant D(t)^{(l-1) / l}\left(\int_{\Omega}|u|^{2 l} \tilde{\rho} \mathrm{d} \mu_{t}\right)^{1 / l} \\
& \leqslant D(t)^{(l-1) / l}\left(\frac{1}{(4 \pi(s-t))^{(d-1) / 2}} \int_{\Omega}|u|^{2 l} \eta \mathrm{d} \mu_{t}\right)^{1 / l},
\end{aligned}
$$

where we choose $l$ so that $2 l=p(d-1) /(d-p)(l>1$ for $(d+2) / 2<p<d)$. Then we apply Theorem 2.1 to the measure $\mu_{t}$ and $\phi=|u| \tilde{\eta}$, where the condition on the density ratio follows from 3.37). $\tilde{\eta}$ is another suitable cut-off function so that $\tilde{\eta}=1$ on $\{\eta \neq 0\}$. From (3.78) we then obtain

$$
\int_{\Omega} \tilde{\rho}|u|^{2} \mathrm{~d} \mu_{t} \leqslant \frac{c_{17}(d, p) D(t)}{(s-t)^{(d-p) / p}}\|u\|_{W^{1, p}(\Omega)}^{2} .
$$

After integrating over $\left[t_{0}, t_{1}\right]$ and using the Hölder inequality and $\left(s-t_{0}\right)^{\iota}-\left(s-t_{1}\right)^{\iota} \leqslant\left(t_{1}-t_{0}\right)^{\iota}$ for $0<(2 p-d-2) /(p-2)=\iota<1$, we obtain 3.76.

Proposition 3.12 Assume $d>p>(d+2 / 2)$ and set $c_{19}=3 e^{1 / 4}(4 \pi)^{(d-1) / 2} / \omega_{d-1}$. For $T_{1} \geqslant t_{1}>t_{0} \geqslant 0$ assume $D\left(t_{1}\right)=c_{19} D\left(t_{0}\right)$ and $\sup _{t \in\left[t_{0}, t_{1}\right]} D(t) \leqslant c_{19} D\left(t_{0}\right)$. Then there exists $\epsilon_{5}=\epsilon_{5}\left(c_{1}, c_{2}, c_{3}, D_{0}, E_{0}, T, \gamma\right)$ such that for all $\varepsilon<\epsilon_{5}$, we have

$$
\left(t_{1}-t_{0}\right)^{\frac{2 p-d-2}{p-2}}\left(\int_{t_{0}}^{t_{1}}\|u(\cdot, t)\|_{W^{1, p}(\Omega)}^{p} \mathrm{~d} t\right)^{2 / p} \geqslant\left(c_{18} c_{19}\right)^{-1} .
$$


Proof. Let $y \in \Omega$ and $0<r<1$ be such that $D\left(t_{1}\right)=\frac{1}{\omega_{d-1} r^{d-1}} \mu_{t_{1}}\left(B_{r}(y)\right)$. Set $s=t_{1}+r^{2}$ and use (3.9), 3.76) and 3.71) to obtain

$$
\begin{aligned}
\int_{\Omega} \tilde{\rho}_{(y, s)} \mathrm{d} \mu_{t_{1}} \leqslant & \int_{\Omega} \tilde{\rho}_{(y, s)} \mathrm{d} \mu_{t_{0}}+c_{19} c_{18} D\left(t_{0}\right)\left(t_{1}-t_{0}\right)^{\frac{2 p-d-2}{p-2}}\left(\int_{t_{0}}^{t_{1}}\|u(\cdot, t)\|_{W^{1, p}(\Omega)}^{p} \mathrm{~d} t\right)^{2 / p} \\
& +\left(t_{1}-t_{0}\right) c_{5} E_{0}+c_{16} \varepsilon^{\gamma^{\prime}-\gamma}
\end{aligned}
$$

By the choice of $s=t_{1}+r^{2}$,

$$
\int_{\Omega} \tilde{\rho}_{(y, s)} \mathrm{d} \mu_{t_{1}}=\int_{\Omega} \frac{e^{-|x-y|^{2} /\left(4 r^{2}\right)}}{(4 \pi)^{(d-1) / 2} r^{d-1}} \mathrm{~d} \mu_{t_{1}} \geqslant \frac{e^{-1 / 4}}{(4 \pi)^{(d-1) / 2} r^{d-1}} \mu_{t_{1}}\left(B_{r}(y)\right)=3 D\left(t_{0}\right) .
$$

The last equality follows from the assumption $c_{19} D\left(t_{0}\right)=D\left(t_{1}\right)$. Since $\int_{\Omega} \tilde{\rho}_{(y, s)} \mathrm{d} \mu_{t_{0}} \leqslant D\left(t_{0}\right)$, 3.80) and (3.81) prove

$$
2 D\left(t_{0}\right) \leqslant c_{19} c_{18} D\left(t_{0}\right)\left(t_{1}-t_{0}\right)^{\frac{2 p-d-2}{p-2}}\left(\int_{t_{0}}^{t_{1}}\|u(\cdot, t)\|_{W^{1, p}(\Omega)}^{p} \mathrm{~d} t\right)^{2 / p}+\left(t_{1}-t_{0}\right) c_{5} E_{0}+c_{16} \varepsilon^{\gamma^{\prime}-\gamma} .
$$

Using $D\left(t_{0}\right) \geqslant 1$ and restricting $\varepsilon$ depending only on $\gamma$ and $c_{16}$, and then restricting $t_{1}-t_{0}$ depending only on $c_{6}$ and $E_{0}$ (which depend only on $d, p, c_{3}, T$ and $D_{0}$ in turn), we obtain (3.79) from 3.82.

Proof of Proposition 3.6 Following the result of Proposition 3.12, set $T_{b}>0$ so that

$$
T_{b}^{\frac{2 p-d-2}{p-2}} c_{3}^{2}=\left(c_{18} c_{19}\right)^{-1}
$$

and $c_{b}=c_{19}$. Note that $T_{b}$ depends only on $d, p$, and $c_{3}$ and $c_{b}$ depends only on $d$. Let $T_{1} \leqslant T$ be the first time when the inequality

$$
D(t)<D_{0} c_{b}^{\left[t / T_{b}\right]+1}
$$

fails. On $\left[0, T_{1}\right]$ we have $D(t) \leqslant D_{0} c_{b}^{\left[T / T_{b}\right]+1}$ and we carry out the argument of the present section. Proposition 3.12 with $t_{0}=0$ shows that we have at least $T_{1} \geqslant T_{b}$. If $T_{1} \in\left[T_{b}, 2 T_{b}\right)$, then $D\left(T_{1}\right)=$ $c_{b}^{2} D_{0}$. Thus there exists some $T_{b} \leqslant t_{0}<T_{1}$ such that $D\left(t_{0}\right)=c_{b} D_{0}$ and $t_{1}-t_{0}<T_{b}$. But Proposition 3.12 says that $t_{1}-t_{0} \geqslant T_{b}$, so $T_{1} \geqslant 2 T_{b}$. Continuing in this manner, we conclude that $T_{1}=T$. The argument for $p>d$ is similar and is omitted.

\section{Existence of limit measure and Brakke's inequality}

In this section we prove the existence of limit measures which correctly describes the motion law of phase boundaries.

THEOREM 4.1 Suppose that sequences $\varphi^{\varepsilon_{i}}$ and $u^{\varepsilon_{i}}$ with $\lim _{i \rightarrow \infty} \varepsilon_{i}=0$ satisfy all the assumptions in Theorem 3.1 where $\varepsilon, \varphi_{0}$ and $\mu_{t}$ are replaced by $\varepsilon_{i}, \varphi_{0}^{\varepsilon_{i}}$ and $\mu_{t}^{\varepsilon_{i}}$, respectively. Assume that $c_{1}, c_{2}$, $c_{3}, D_{0}, \gamma$ and $T$ are independent of $i$. In addition assume that $d=2$ or 3 and that

$$
u^{\varepsilon_{i}} \rightarrow u \text { weakly in } L^{p}\left(0, T ;\left(W^{1, p}(\Omega)\right)^{d}\right), \quad u^{\varepsilon_{i}} \rightarrow u \text { strongly in } L^{2}\left(0, T ;\left(L^{2}(\Omega)\right)^{d}\right) .
$$

Then there exists a subsequence (denoted by the same index) and a family $\left\{\mu_{t}\right\}_{0 \leqslant t \leqslant T}$ of measures such that 
(a) $\lim _{i \rightarrow \infty} \mu_{t}^{\varepsilon_{i}}(\phi)=\mu_{t}(\phi)$ for all $t \in[0, T]$ and $\phi \in C(\Omega)$,

(b) $\mu_{t} \in \mathcal{I M}_{d-1}(\Omega)$ for a.e. $t \in[0, T]$,

(c) $u, H \in L^{2}\left(0, T ;\left(L^{2}\left(\mu_{t}\right)\right)^{d}\right)$ where $H(\cdot, t)$ is the generalized mean curvature of $\mu_{t}$,

(d) for any $0 \leqslant t_{1}<t_{2} \leqslant T$,

$$
\lim _{i \rightarrow \infty} \frac{1}{\sigma} \int_{t_{1}}^{t_{2}} \int_{\Omega} \varepsilon_{i} u^{\varepsilon_{i}} \cdot \nabla \varphi^{\varepsilon_{i}}\left(-\Delta \varphi^{\varepsilon_{i}}+\frac{W^{\prime}\left(\varphi^{\varepsilon_{i}}\right)}{\varepsilon_{i}^{2}}\right) \mathrm{d} x \mathrm{~d} t=\int_{t_{1}}^{t_{2}} \int_{\Omega} H \cdot u \mathrm{~d} \mu_{t} \mathrm{~d} t
$$

(e) for any $\phi \in C^{2}\left(\Omega ; \mathbb{R}^{+}\right)$and $0 \leqslant t_{1}<t_{2} \leqslant T$,

$$
\mu_{t_{2}}(\phi)-\mu_{t_{1}}(\phi) \leqslant \int_{t_{1}}^{t_{2}} \mathcal{B}\left(\mu_{t}, u(\cdot, t), \phi\right) \mathrm{d} t .
$$

We prove Theorem 4.1 through the next four propositions. For notational simplicity we often drop the dependence on $i$ from $\varphi^{\varepsilon_{i}}$ and $\mu_{t}^{\varepsilon_{i}}$ when there should be no confusion in the proofs. Without loss of generality we assume that $\varepsilon_{i}<\epsilon_{1}$ so that we have (3.8) for $\mu_{t}^{\varepsilon_{i}}$ for all $t \in[0, T]$. As a result we also have (3.38) with $T$ in place of $T_{1}$. We first show

PROPOSITION 4.2 For any fixed $\phi \in C^{2}\left(\Omega ; \mathbb{R}^{+}\right)$the function

$$
\mu_{t}^{\varepsilon_{i}}(\phi)-c_{20} t-c_{21} \int_{0}^{t}\left(\int_{\Omega}\left|\nabla u^{\varepsilon_{i}}(x, s)\right|^{2} \mathrm{~d} x\right)^{1 / 2} \mathrm{~d} s
$$

of $t$ is decreasing on [0,T]. Here $c_{20}$ and $c_{21}$ depend only on $\phi, d, p, T, D_{0}$ and $c_{3}$.

Proof. By integration by parts and 3.1), one obtains

$$
\frac{\mathrm{d}}{\mathrm{d} t} \int_{\Omega} \phi \mathrm{d} \mu_{t}^{\varepsilon} \leqslant \int_{\Omega}\left(\phi\left|u^{\varepsilon}\right|^{2}+\frac{|\nabla \phi|^{2}}{\phi}\right) \mathrm{d} \mu_{t}^{\varepsilon} .
$$

The second term of (4.5) can be bounded in terms of $E_{0}$ and a $\phi$-dependent constant. Use Theorem 2.1 (with $p=1$ ) and Theorem 3.1 to estimate the first term as

$$
\int_{\Omega} \phi\left|u^{\varepsilon}\right|^{2} \mathrm{~d} \mu_{t}^{\varepsilon} \leqslant c_{M Z} c_{4} \int_{\Omega}\left|\nabla\left(\phi\left|u^{\varepsilon}\right|^{2}\right)\right| \mathrm{d} x \leqslant c_{23}\left(c_{3}^{p}+c_{3}^{p / 2}\left(\int_{\Omega}\left|\nabla u^{\varepsilon}\right|^{2}\right)^{1 / 2}\right) .
$$

Here we used (3.6) to estimate the $L^{2}$-norm of $u$. By 4.5) and 4.6, we conclude that 4.4 holds with suitable $c_{20}$ and $c_{21}$.

Next we prove the existence of a convergent subsequence $\left\{\mu_{t}^{\varepsilon_{i}}\right\}$ and the limit measure $\mu_{t}$ for all $t$.

Proposition 4.3 There exist a Radon measure $\mu_{t}$ and a subsequence $\left\{\mu_{t}^{\varepsilon_{i}}\right\}_{i} \geqslant 1$ such that for any $t \in[0, T]$,

$$
\mu_{t}^{\varepsilon_{i}} \rightarrow \mu_{t} \quad \text { as Radon measures. }
$$

Proof. Fix $\phi \in C^{2}\left(\Omega ; \mathbb{R}^{+}\right)$. By Schwarz's inequality and the assumption $p>(d+2) / 2 \geqslant 2$,

$$
\int_{t_{1}}^{t_{2}}\left(\int_{\Omega}\left|\nabla u^{\varepsilon_{i}}\right|^{2} \mathrm{~d} x\right)^{1 / 2} \mathrm{~d} s \leqslant\left(t_{2}-t_{1}\right)^{(p-1) / p}\left\|u^{\varepsilon_{i}}\right\|_{L^{p}\left(t_{1}, t_{2} ;\left(W^{1, p}(\Omega)\right)\right)^{d}} \leqslant\left(t_{2}-t_{1}\right)^{(p-1) / p} c_{3},
$$


which implies that the last term in (4.4) is uniformly bounded in Hölder continuous norm with exponent $(p-1) / p$. Thus by the Ascoli-Arzelà compactness theorem, there exists a subsequence which converges uniformly on $[0, T]$. By Proposition 4.2 we may then choose a subsequence such that $\mu_{t}^{\varepsilon_{i}}(\phi)$ converges on a co-countable set $B(\phi) \subset[0, T]$. Now choose a countable set $\left\{\phi_{k}\right\} \subset$ $C^{2}\left(\Omega ; \mathbb{R}^{+}\right)$which is dense in $C\left(\Omega ; \mathbb{R}^{+}\right)$. We can choose a subsequence such that $\mu_{t}^{\varepsilon_{i}}\left(\phi_{k}\right)$ converges on a co-countable set $B=\bigcap_{k} B\left(\phi_{k}\right)$. Define $\mu_{t}\left(\phi_{k}\right)=\lim _{i \rightarrow \infty} \mu_{t}^{\varepsilon_{i}}\left(\phi_{k}\right)$ for $t \in B$. By density argument, we conclude that $\mu_{t}(\phi)=\lim _{i \rightarrow \infty} \mu_{t}^{\varepsilon_{i}}(\phi)$ for all $\phi \in C(\Omega)$ for $t \in B$. Since the complement of $B$ is countable, we may further choose a subsequence so that $\mu_{t}\left(\phi_{k}\right)$ converges on $[0, T] \backslash B$ as well, and so does $\mu_{t}(\phi)$ for all $\phi \in C(\Omega)$.

Proposition 4.4 For a.e. $t \in[0, T], \mu_{t}$ is integral and

$$
\int_{0}^{T} \int_{\Omega}|H|^{2} \mathrm{~d} \mu_{t} \mathrm{~d} t \leqslant \liminf _{i \rightarrow \infty} \frac{1}{\sigma} \int_{0}^{T} \int_{\Omega} \varepsilon_{i}\left(\Delta \varphi^{\varepsilon_{i}}-\frac{W^{\prime}\left(\varphi^{\varepsilon_{i}}\right)}{\varepsilon_{i}^{2}}\right)^{2} \mathrm{~d} x \mathrm{~d} t .
$$

Proof. By (3.38), $d=2,3$ and Fatou's Lemma, the conditions to apply Theorem 2.6 are satisfied for a.e. $t \in[0, T]$. The claim is a direct consequence.

Proposition 4.5 We have 4.2 and 4.3.

Proof. First note that by arguing as in Section 2.2, the right-hand sides of (4.2) and (4.3) are welldefined since $u \in L^{p}\left(0, T ;\left(W^{1, p}(\Omega)\right)^{d}\right)$ is enough for them. Compute $\frac{\mathrm{d}}{\mathrm{d} t} \mu_{t}^{\varepsilon_{i}}(\phi)$ using 3.1 and integrate over $t \in\left[t_{1}, t_{2}\right]$ to obtain

$$
\begin{aligned}
\mu_{t_{2}}^{\varepsilon}(\phi)-\mu_{t_{1}}^{\varepsilon}(\phi)= & \frac{1}{\sigma} \int_{t_{1}}^{t_{2}} \int_{\Omega}\left(-\varepsilon \phi\left(\Delta \varphi^{\varepsilon}-\frac{W^{\prime}}{\varepsilon^{2}}\right)^{2}-\varepsilon \nabla \phi \cdot \nabla \varphi^{\varepsilon}\left(\Delta \varphi^{\varepsilon}-\frac{W^{\prime}}{\varepsilon^{2}}\right)\right. \\
& \left.+\varepsilon \phi\left(\Delta \varphi^{\varepsilon}-\frac{W^{\prime}}{\varepsilon^{2}}\right) u^{\varepsilon} \cdot \nabla \varphi^{\varepsilon}+\varepsilon\left(\nabla \varphi^{\varepsilon} \cdot \nabla \phi\right)\left(u^{\varepsilon} \cdot \nabla \varphi^{\varepsilon}\right)\right) \mathrm{d} x \mathrm{~d} t .
\end{aligned}
$$

The left-hand side converges to $\mu_{t_{2}}(\phi)-\mu_{t_{1}}(\phi)$ as $\varepsilon \rightarrow 0$ by Proposition 4.3 . By exactly the same argument as in [24, Lemma 3.2], we have

$$
\begin{aligned}
\limsup _{i \rightarrow \infty} \frac{1}{\sigma} \int_{t_{1}}^{t_{2}} \int_{\Omega}\left(-\varepsilon \phi\left(\Delta \varphi^{\varepsilon}-\frac{W^{\prime}}{\varepsilon^{2}}\right)^{2}-\varepsilon \nabla \phi\right. & \left.\cdot \nabla \varphi^{\varepsilon}\left(\Delta \varphi^{\varepsilon}-\frac{W^{\prime}}{\varepsilon^{2}}\right)\right) \mathrm{d} x \mathrm{~d} t \\
& \leqslant \int_{t_{1}}^{t_{2}} \int_{\Omega}\left(-\phi|H|^{2}+\nabla \phi \cdot H\right) \mathrm{d} \mu_{t} \mathrm{~d} t
\end{aligned}
$$

for the first two terms of the right-hand side of (4.7). For the third term, we show that it converges to $\int_{t_{1}}^{t_{2}} \int \phi H \cdot u \mathrm{~d} \mu_{t} \mathrm{~d} t$. Let $\left\{g_{i}\right\} \in C^{\infty}\left(\Omega \times[0, T] ; \mathbb{R}^{d}\right)$ be a sequence of functions such that $\lim _{i \rightarrow \infty}\left\|u-g_{i}\right\|_{L^{2}\left(0, T ;\left(W^{1,2}(\Omega)\right)^{d}\right)}=0$. Here we have a sequence $u^{\varepsilon}$ converging strongly to $u$ and $\nabla u^{\varepsilon}$ converging weakly to $\nabla u$ both in $L^{2}(\Omega \times[0, T])$. We interpolate as

$$
\begin{aligned}
\left|\frac{1}{\sigma} \int_{t_{1}}^{t_{2}} \int_{\Omega} \varepsilon \phi\left(\Delta \varphi^{\varepsilon}-\frac{W^{\prime}}{\varepsilon^{2}}\right) u^{\varepsilon} \cdot \nabla \varphi^{\varepsilon} \mathrm{d} x \mathrm{~d} t+\int_{t_{1}}^{t_{2}} \int_{\Omega} \phi u \cdot H \mathrm{~d} \mu_{t} \mathrm{~d} t\right| \\
\leqslant\left|\frac{1}{\sigma} \int_{t_{1}}^{t_{2}} \int_{\Omega} \varepsilon \phi\left(\Delta \varphi^{\varepsilon}-\frac{W^{\prime}}{\varepsilon^{2}}\right)\left(u^{\varepsilon}-g_{i}\right) \cdot \nabla \varphi^{\varepsilon} \mathrm{d} x \mathrm{~d} t\right|+\left|\int_{t_{1}}^{t_{2}} \int_{\Omega} \phi\left(g_{i}-u\right) \cdot H \mathrm{~d} \mu_{t} \mathrm{~d} t\right| \\
\quad+\left|\frac{1}{\sigma} \int_{t_{1}}^{t_{2}} \int_{\Omega} \varepsilon \phi\left(\Delta \varphi^{\varepsilon}-\frac{W^{\prime}}{\varepsilon^{2}}\right) g_{i} \cdot \nabla \varphi^{\varepsilon} \mathrm{d} x \mathrm{~d} t+\int_{t_{1}}^{t_{2}} \int_{\Omega} \phi g_{i} \cdot H \mathrm{~d} \mu_{t} \mathrm{~d} t\right|
\end{aligned}
$$


Using varifold convergence, the third term on the right converges to 0 as $\varepsilon \rightarrow 0$. The first and second terms are bounded by $C\left\|u^{\varepsilon}-g_{i}\right\|_{L^{2}(\Omega \times[0, T])}$ and $C\left\|u-g_{i}\right\|_{L^{2}(\Omega \times[0, T])}$ where $C$ depends on $c_{4}$, $c_{M Z}, E_{0}$ and $L^{2}\left(0, T ;\left(W^{1,2}(\Omega)\right)^{d}\right)$-norms of $g_{i}, u^{\varepsilon}$ and $u$, which are all uniformly bounded. Thus (4.9) goes to 0 as $\varepsilon \rightarrow 0$. A similar argument using approximation and varifold convergence shows that

$$
\lim _{\varepsilon \rightarrow 0} \frac{1}{\sigma} \int_{t_{1}}^{t_{2}} \int \varepsilon\left(\nabla \varphi^{\varepsilon} \cdot \nabla \phi\right)\left(u^{\varepsilon} \cdot \nabla \varphi^{\varepsilon}\right) \mathrm{d} x \mathrm{~d} t=\int_{t_{1}}^{t_{2}} \int(\nabla \phi \cdot n)(u \cdot n) \mathrm{d} \mu_{t} \mathrm{~d} t .
$$

Combining (4.7)- 4.10) and rearranging terms, we obtain 4.3).

The proof of Theorem 4.1 is now complete except for $u \in L^{2}\left(0, T ; L^{2}\left(\mu_{t}\right)^{d}\right)$. But this follows easily from 3.8$)$ and $(2.4)$.

REMARK 4.6 We note that the proof for (4.3) goes through even when $\phi \in C^{3}(\Omega \times[0, T])$ depends on $t$, except that we have the extra term $\int_{t_{1}}^{t_{2}} \int_{\Omega} \frac{\partial \phi}{\partial t} \mathrm{~d} \mu_{t}$ in (4.7). This gives the proof of 2.7. Theorem 2.3 b) also follows from (3.8) and Proposition 4.3 .

THEOREM 4.7 Under the same assumptions as in Theorem 4.1 we have a subsequence $\left\{\varphi^{\varepsilon_{i}}\right\}$ and a function $\varphi \in B V(\Omega \times[0, T]) \cap L^{\infty}(0, T ; B V(\Omega)) \cap C^{1 / 2}\left([0, T] ; L^{1}(\Omega)\right)$ such that for all $t \in[0, T]$,

(i) $\lim _{i \rightarrow \infty}\left\|\varphi^{\varepsilon_{i}}(\cdot, t)-\varphi(\cdot, t)\right\|_{L^{1}(\Omega)}=0$,

(ii) $\varphi(\cdot, t)= \pm 1$ a.e. on $\Omega$,

(iii) $\operatorname{spt}\left|\nabla \chi_{\{\varphi(\cdot, t)=1\}}\right| \subset \operatorname{spt} \mu_{t}$.

Proof. For the convergence of $\varphi^{\varepsilon}$, let

$$
\Phi(s)=\frac{1}{\sigma} \int_{-1}^{s} \sqrt{2 W(y)} \mathrm{d} y, \quad w^{\varepsilon}=\Phi \circ \varphi^{\varepsilon} .
$$

Note that $\Phi(1)=1$. Then

$$
\left|\nabla w^{\varepsilon}\right|=\frac{1}{\sigma}\left|\nabla \varphi^{\varepsilon}\right| \sqrt{2 W\left(\varphi^{\varepsilon}\right)} \leqslant \frac{1}{\sigma}\left(\frac{\varepsilon\left|\nabla \varphi^{\varepsilon}\right|^{2}}{2}+\frac{W\left(\varphi^{\varepsilon}\right)}{\varepsilon}\right) .
$$

Therefore by 3.38,

$$
\int_{\Omega}\left|\nabla w^{\varepsilon}(\cdot, t)\right| \mathrm{d} x \leqslant \frac{1}{\sigma} \int_{\Omega}\left(\frac{\varepsilon\left|\nabla \varphi^{\varepsilon}\right|^{2}}{2}+\frac{W\left(\varphi^{\varepsilon}\right)}{\varepsilon}\right) \mathrm{d} x \leqslant E_{0}
$$

for all $t \in[0, T]$. By an argument using Theorem 2.1 the upper density ratio bound (3.8) and 3.38) we also deduce that

$$
\begin{aligned}
\int_{0}^{T} \int_{\Omega} \mid & \left|\frac{\partial w^{\varepsilon}}{\partial t}\right| \mathrm{d} x \mathrm{~d} t \leqslant \frac{1}{\sigma} \int_{0}^{T} \int_{\Omega}\left(\frac{\varepsilon\left|\frac{\partial \varphi^{\varepsilon}}{\partial t}\right|^{2}}{2}+\frac{W\left(\varphi^{\varepsilon}\right)}{\varepsilon}\right) \mathrm{d} x \mathrm{~d} t \\
& \leqslant \frac{1}{\sigma} \int_{0}^{T} \int_{\Omega} \varepsilon\left\{\left(u^{\varepsilon} \cdot \nabla \varphi^{\varepsilon}\right)^{2}+\left(\Delta \varphi^{\varepsilon}-\frac{W^{\prime}\left(\varphi^{\varepsilon}\right)}{\varepsilon^{2}}\right)^{2}\right\} \mathrm{d} x \mathrm{~d} t+\frac{1}{\sigma} \int_{0}^{T} \int_{\Omega} \frac{W\left(\varphi^{\varepsilon}\right)}{\varepsilon} \mathrm{d} x \mathrm{~d} t \\
& \leqslant c_{M Z} c_{3} c_{4}+E_{0}+T E_{0} .
\end{aligned}
$$


Thus (4.11) and (4.12) show that $\left\{w^{\varepsilon}\right\}$ is bounded in $B V(\Omega \times[0, T])$. By the standard compactness theorem, there exists a subsequence and $w \in B V(\Omega \times[0, T])$ such that $w^{\varepsilon_{i}}$ converges to $w$ strongly in $L^{1}(\Omega \times[0, T])$ and a.e. pointwise. Thus by defining $f=\Phi^{-1} \circ w$ we have

$$
\varphi^{\varepsilon_{i}} \rightarrow \varphi \quad \text { a.e. in } \Omega \times[0, T]
$$

and by combining this property and $\left|\varphi^{\varepsilon_{i}}\right| \leqslant 1$ we obtain

$$
\varphi^{\varepsilon_{i}} \rightarrow \varphi \quad \text { in } L^{1}(\Omega \times[0, T]) .
$$

The energy bound also gives $\varphi= \pm 1$ a.e. on $\Omega \times[0, T]$. For a.e. $0 \leqslant t_{1}<t_{2} \leqslant T$,

$$
\begin{aligned}
\int_{\Omega}\left|w\left(\cdot, t_{1}\right)-w\left(\cdot, t_{2}\right)\right| \mathrm{d} x & =\lim _{i \rightarrow \infty} \int_{\Omega}\left|w^{\varepsilon_{i}}\left(\cdot, t_{1}\right)-w^{\varepsilon_{i}}\left(\cdot, t_{2}\right)\right| \mathrm{d} x \leqslant \liminf _{i \rightarrow \infty} \int_{\Omega} \int_{t_{1}}^{t_{2}}\left|\frac{\partial w^{\varepsilon_{i}}}{\partial t}\right| \mathrm{d} t \mathrm{~d} x \\
& \leqslant \liminf _{i \rightarrow \infty} \frac{1}{\sigma} \int_{\Omega} \int_{t_{1}}^{t_{2}}\left(\frac{\varepsilon_{i}\left|\partial \varphi^{\varepsilon_{i}} / \partial t\right|^{2}}{2} \sqrt{t_{2}-t}+\frac{W\left(\varphi^{\varepsilon_{i}}\right)}{\varepsilon_{i} \sqrt{t_{2}-t}}\right) \mathrm{d} t \mathrm{~d} x
\end{aligned}
$$

By a similar argument to 4.12, we obtain

$$
\int_{\Omega}\left|w\left(\cdot, t_{1}\right)-w\left(\cdot, t_{2}\right)\right| \mathrm{d} x \leqslant\left(c+E_{0}\right) \sqrt{t_{2}-t_{1}} .
$$

Consequently, $\varphi \in B V(\Omega \times[0, T]) \cap C^{1 / 2}\left([0, T] ; L^{1}(\Omega)\right)$. The continuity of $w(\cdot, t)$ in $L^{1}(0, T)$ gives a well-defined $w(\cdot, t)$ for all $t \in[0, T]$ and one can show that $w^{\varepsilon_{i}}(\cdot, t) \rightarrow w(\cdot, t)$ strongly in $L^{1}(\Omega)$ for all $t \in[0, T]$ (not just a.e. $t \in[0, T]$ ) and the same for $\varphi^{\varepsilon_{i}} \rightarrow \varphi$ with $\varphi(\cdot, t)= \pm 1$ a.e. on $\Omega$. Finally for any $\phi \in C(\Omega)$ and $t \in[0, T]$,

$$
\begin{aligned}
\int_{\Omega} \phi \mathrm{d}|\nabla \chi\{\varphi(\cdot, t)=1\}|=\int_{\Omega} \phi|\nabla w| & \leqslant \liminf _{i \rightarrow \infty} \int_{\Omega}\left|\nabla w^{\varepsilon_{i}}\right| \phi \mathrm{d} x \\
& \leqslant \lim _{i \rightarrow \infty} \frac{1}{\sigma} \int_{\Omega}\left(\frac{\varepsilon_{i}\left|\nabla \varphi^{\varepsilon_{i}}\right|^{2}}{2}+\frac{W\left(\varphi^{\varepsilon_{i}}\right)}{\varepsilon_{i}}\right) \phi \mathrm{d} x=\int_{\Omega} \phi \mathrm{d} \mu_{t}
\end{aligned}
$$

which yields (iii)

\section{Proof of Theorem 2.2}

The proof of the main theorem can be completed by suitably fitting the given data $\Gamma(0)$ and $u$ so that the approximate data satisfy the assumptions of Theorem 3.1. We give a proof for $\Omega^{+}(0)$ belonging to the class described in Remark 2.5. For such $\Omega^{+}(0) \subset \Omega$ suppose $\Omega^{+}(0)^{i}$ are approximating domains which have $C^{3}$ boundary $\Gamma(0)^{i}$. Let $d^{i}(x)$ be the signed distance function to $\Gamma(0)^{i}$ so that $d^{i}(x)>0$ on $\Omega^{+}(0)^{i}$. Choose $b^{i}>0$ so that $d^{i}$ is a $C^{3}$ function on the $b^{i}$-neighborhood of $\Gamma(0)^{i}$. Let $h^{i} \in C^{\infty}(\mathbb{R})$ be a function such that $h^{i}$ is increasing, $h^{i}(s)=s$ for $0 \leqslant s \leqslant b^{i} / 4$ and $h^{i}(s)=b^{i} / 2$ for $b^{i} / 2<s$, and define $h^{i}(s)=-h^{i}(-s)$ for $s<0$. Then define $\tilde{d}^{i}(x)=h^{i}\left(d^{i}(x)\right)$. We then choose a sequence $\varepsilon_{i}$ so that $\lim _{i \rightarrow \infty} \varepsilon_{i} / b^{i}=0$ and define

$$
\varphi_{0}^{\varepsilon_{i}}(x)=\tanh \left(\tilde{d}^{i}(x) / \varepsilon_{i}\right)
$$


Then $\varphi_{0}^{\varepsilon_{i}} \in C^{3}(\Omega)$. We may also choose $\varepsilon_{i}$ so that $(3.3)$ holds for all $i$. By using a well-known property of (5.1) and choosing smaller $\varepsilon_{i}$ 's if necessary, we may assume that

$$
\lim _{i \rightarrow \infty} \int_{\Omega}\left|\left(\varphi_{0}^{\varepsilon_{i}}+1\right) / 2-\chi_{\Omega^{+}(0)}\right| \mathrm{d} x=0 \text { and } \lim _{i \rightarrow \infty} \frac{1}{\sigma}\left(\frac{\varepsilon_{i}\left|\nabla \varphi_{0}^{\varepsilon_{i}}\right|^{2}}{2}+\frac{W\left(\varphi_{0}^{\varepsilon_{i}}\right)}{\varepsilon_{i}}\right) \mathrm{d} x=\left|\nabla \chi_{\Omega^{+}(0)}\right|
$$

as measures by 2.8. As the initial data in Theorem 3.1 $\varphi_{0}^{\varepsilon_{i}}$ satisfies 3.3, 3.4 and $D(0)$ is uniformly bounded due to (2.8), and thus we have some $D_{0}$ independent of $i$. Thus (3.7) is also satisfied.

Set $T_{i}=i$ for all $i \in \mathbb{N}$. For $u \in L_{\text {loc }}^{p}\left([0, \infty) ;\left(W^{1, p}(\Omega)\right)^{d}\right)$, by a density argument, we may choose a sequence $u^{i} \in C^{\infty}\left(\Omega \times\left[0, T_{i}\right] ; \mathbb{R}^{d}\right)$ with $\lim _{i \rightarrow \infty}\left\|u^{i}-u\right\|_{L^{p}\left(0, T_{i} ;\left(W^{1, p}(\Omega)\right)^{d}\right)}=0$. We then associate to each $u^{i}$ some small enough $\varepsilon_{j_{i}}$ so that $\left\|\varepsilon_{j_{i}}^{\gamma} u^{i}, \varepsilon_{j_{i}}^{1+\gamma} \nabla u^{i}\right\|_{L^{\infty}\left(\Omega \times\left[0, T_{i}\right]\right)} \leqslant 1$ and so that $\varepsilon_{j_{i}}<\epsilon_{1}$ where $\epsilon_{1}$ depends on quantities corresponding to $T_{i}$ in Theorem 3.1. We then renumber $\varepsilon_{j_{i}}$ as $\varepsilon_{i}$.

Now we solve

$$
\begin{cases}\frac{\partial \varphi^{\varepsilon_{i}}}{\partial t}+u^{i} \cdot \nabla \varphi^{\varepsilon_{i}}=\Delta \varphi^{\varepsilon_{i}}-\frac{W^{\prime}\left(\varphi^{\varepsilon_{i}}\right)}{\varepsilon_{i}^{2}} & \text { on } \Omega \times\left[0, T_{i}\right], \\ \varphi^{\varepsilon_{i}}(x, 0)=\varphi_{0}^{\varepsilon_{i}}(x) & \text { on } \Omega .\end{cases}
$$

The existence of a smooth solution is standard. Since $u^{i}$ satisfies (3.5) and converges to $u$ in the sense required in Theorem 4.1, the solution $\varphi^{\varepsilon_{i}}$ satisfies all the assumptions of Theorems 4.1 and 4.7 for each arbitrarily fixed $T>0$. By extracting a diagonal subsequence, this completes the proof of Theorem 2.2 a)\&(b). The conclusions on the initial data in (a) and (b) follow easily from 5.2 . We are left with the proof of (c). Since $\mu_{t}$ is integral, we only need to prove that $\left\{\theta_{t} \geqslant 2\right\}$ has measure zero for a.e. $0 \leqslant t \leqslant T_{1}$ for a suitable $T_{1}$. Suppose the converse. Then we have some point $x$ with $\theta_{t}(x)=N \geqslant 2$ and $\lim _{r \rightarrow 0} \mu_{t}\left(B_{r}(x)\right) /\left(\omega_{d-1} r^{d-1}\right)=N$. Then after a computation as in 3.35) we find that

$$
\lim _{r \rightarrow 0} \int_{\Omega} \tilde{\rho}_{\left(x, t+r^{2}\right)} \mathrm{d} \mu_{t}=N .
$$

By (2.9) we have $\int_{\Omega} \tilde{\rho}_{(x, t)} \mathrm{d} \mu_{0} \leqslant 1+o(1)$ as $t \rightarrow 0$. By letting $\varepsilon \rightarrow 0$ in 3.80) with $t_{0}=0$ and $t_{1}=t$, we would then have $N \leqslant 1+o(1)$, where the smallness depends on $c_{3}, \Omega^{+}(0)$ and $p$ ultimately. This gives a contradiction and $\mu_{t}$ has unit density for $t \leqslant T_{1}$. To prove the last claim, $\left|\nabla \chi_{\{\varphi(\cdot, t)=1\}}\right|=\mu_{t}$ a.e. $t \in\left[0, T_{1}\right]$, suppose $\neq$ for a contradiction for a positive measure. We may assume that $\mu_{t}$ is integral and has unit density. Then we may assume that $\mu_{t}=\mathcal{H}^{d-1}\left\lfloor\Gamma(t)\right.$ with $(d-1)$-rectifiable set $\Gamma(t)$. Since $\left|\nabla \chi_{\{\varphi(\cdot, t)=1\}}\right| \ll \mu_{t}$ by (b-3), having $\neq$ means that $\mathcal{H}^{d-1}\left(\Gamma(t) \backslash \partial^{*}\{\varphi(\cdot, t)=1\}\right)>0$. Then there is a density point which does not belong to the reduced boundary and also the density is 1 there. In addition we may assume that $\liminf _{i \rightarrow \infty} \varepsilon_{i} \int_{\Omega}\left(\Delta \varphi^{\varepsilon_{i}}-W^{\prime} / \varepsilon_{i}^{2}\right)^{2} \mathrm{~d} x<\infty$ for such $t$. Then one can check the proof of integrality of the limiting varifold in [23, Proposition 5.2] to see that $x \in \partial^{*}\{\varphi(\cdot, t)=1\}$. This contradicts the assumption and completes the proof of Theorem 2.2 .

\section{Remarks}

Generalizations. In this paper we worked with $\Omega=\mathbb{T}^{d}$. It is obvious that the claims and proofs are identical for a flat torus with any size. For $\Omega=\mathbb{R}^{d}$, suppose we have some $R>0$ such that 
$\Gamma(0) \subset B_{R}(0)$ and $u=0$ on the complement of $B_{R}(0)$ for all $t>0$. Then the same conclusions can be deduced by considering a flat torus large enough to include $B_{R}(0)$. One can prove by using an argument as in [5] that the support of $\mu_{t}$ remains in $B_{R}(0)$ for all $t>0$ by using a suitable test function. For $d>3$, we expect that the approach of [14] and [27] should go through since we have the key estimate (3.8) though we may be missing some technical difficulties.

Critical power. As for the power $p, p=(d+2) / 2$ is a critical value in the sense that

$$
\int_{0}^{\infty} \int_{\mathbb{R}^{d}}|\nabla u|^{p} \mathrm{~d} x \mathrm{~d} t
$$

is invariant under the natural scaling $\tilde{x}=\lambda x, \tilde{t}=\lambda^{2} t$ and $\tilde{u}=\lambda^{-1} u$. It is not clear if the density upper ratio bound (3.8) holds for the critical case. It is not clear either if the measure $\mu_{t}(\Omega)$ remains finite in that case. Nothing seems to be known for this problem as far as we know.

Coupled problems. In case $u$ satisfies additional equations (such as Navier-Stokes or just incompressibility condition div $\mathrm{u}=0$ for example) we may expect the same type of conclusions to hold under relaxed conditions such as a smaller exponent for $W^{1, p}$. We should mention the work of Plotnikov [22] who considered the kinematic condition $V_{\Gamma}=(u \cdot n) n$ for the two-phase fluid problem in $d=2$ and $p>2$ which comes naturally with the interface length control due to coupling. He obtained the existence of a rectifiable varifold which satisfies the motion law. He used incompressibility to deduce the rectifiability of the varifold even though there is no control of mean curvature.

\section{Acknowledgements}

Y. T. is partially supported by JSPS Grant-in-aid for scientific research (B) 80296748.

\section{REFERENCES}

1. Allard, W. On the first variation of a varifold. Ann. of Math. 95 (1972), 417-491. Zbl 0252.49028 MR 0307015

2. Allen, S. M., \& CAHn, J. W. A macroscopic theory for antiphase boundary motion and its application to antiphase domain coarsening. Acta Metal. 27 (1979) 1085-1095.

3. Almgren, F., TAYlor, J. E., \& WANG, L.-H. Curvature-driven flows: a variational approach. SIAM J. Control Optim. 31 (1993), 387-438. Zbl 0783.35002 MR 1205983

4. Barles, G., \& Souganidis, P. E. A new approach to front propagation problem: theory and applications. Arch. Ration. Mech. Anal. 141 (1998), 237-296. Zbl 0904.35034 MR 1617291

5. BRAKKe, K. The Motion of a Surface by its Mean Curvature. Princeton Univ. Press, Princeton, NJ (1978). Zbl 0386.53047 MR 0485012

6. Chen, Y.-G., Giga, Y., \& Goto, S. Uniqueness and existence of viscosity solutions of generalized mean curvature flow equations. J. Differential Geom. 33 (1991), 749-786. Zbl 0696.35087||MR 1100211

7. CHEN, Y., \& STRUwe, M. Existence and partial regularity results for the heat flow for harmonic maps. Math. Z. 201 (1989), 83-103. Zbl 0652.58024 MR 0990191

8. ECKER, K. On regularity for mean curvature flow of hypersurfaces. Calc. Var. Partial Differential Equations 3 (1995), 107-126. Zbl 0814.53004 MR 1384839

9. ECKER, K. Regularity Theory for Mean Curvature Flow. Progr. Nonlinear Differential Equations Appl. 57, Birkhäuser Boston, Boston, MA (2004). Zbl 1058.53054 MR 2024995 
10. Evans, L. C., \& GariePy, R. F. Measure Theory and Fine Properties of Functions. Stud. Adv. Math., CRC Press (1992). Zbl 0804.28001 MR 1158660

11. Evans, L. C., \& SpRUCK, J. Motion of level sets by mean curvature I. J. Differential Geom. 33 (1991), 635-681. Zbl 0726.53029 MR 1100206

12. Huisken, G. Asymptotic behavior for singularities of the mean curvature flow. J. Differential Geom. 31 (1990), 285-299. Zbl 0694.53005 MR 1030675

13. Hutchinson, J. E., \& Tonegawa, Y. Convergence of phase interfaces in the van der WaalsCahn-Hilliard theory. Calc. Var. Partial Differential Equations 10 (2000), 49-84. Zbl 1070.49026 MR 1803974

14. Ilmanen, T. Convergence of the Allen-Cahn equation to Brakke's motion by mean curvature. J. Differential Geom. 38 (1993), 417-461. Zbl 0784.53035 MR 1237490

15. Ladyzhenskaya, O. A., Solonnikov, V. A., \& URAl'TSEVA, N. N. Linear and Quasilinear Equations of Parabolic Type. Transl. Math. Monogr. 23, Amer. Math. Soc. (1968). Zbl 0164.12302 MR 0241822

16. LiU, C., \& Walkington, N. J. An Eulerian description of fluids containing visco-hyperelastic particles. Arch. Ration. Mech. Anal. 159 (2001), 229-252. Zbl 1009.76093 MR 1857673

17. Luckhaus, S., \& Sturzenhecker, T. Implicit time discretization for the mean curvature flow equation. Calc. Var. Partial Differential Equations 3 (1995), 253-271. Zbl 0821.35003 MR 1386964

18. MAEKAWA, Y. On a free boundary problem for viscous incompressible flows. Interfaces Free Bound. 9 (2007), 549-589. Zbl 1132.76303 MR 2358216

19. Meyers, N. G., \& ZIEMER, W. P. Integral inequalities of Poincaré and Wirtinger type for BV functions. Amer. J. Math. 99 (1977), 1345-1360. Zbl 0416.46025 MR 0507433

20. Mugnai, L., \& Röger, M. The Allen-Cahn action functional in higher dimensions. Interfaces Free Bound. 10 (2008), 45-78. Zbl pre05302266 MR 2383536

21. Mugnai, L., \& RöGer, M. Convergence of perturbed Allen-Cahn equations to forced mean curvature flow. arXiv:0902.1816

22. Plotnikov, P. I. Generalized solutions to a free boundary problem of motion of a non-Newtonian fluid. Siberian Math. J. 34 (1993), 704-716. Zbl 0814.76007| MR 1248797

23. Röger, M., \& SchätZle, R. On a modified conjecture of De Giorgi. Math. Z. 254 (2006), 675-714. Zbl 1126.49010 MR 2253464

24. SAto, N. A simple proof of convergence of the Allen-Cahn equation to Brakke's motion by mean curvature. Indiana Univ. Math. J. 57 (2008), 1743-1751. Zbl 1160.35444 MR 2440879

25. Simon, L. Lectures on Geometric Measure Theory. Proc. Centre Math. Anal. Austral. Nat. Univ. 3 (1983). Zbl 0546.49019 MR 0756417

26. SONER, H. M. Convergence of the phase-field equations to the Mullins-Sekerka problem with kinetic undercooling. Arch. Ration. Mech. Anal. 131 (1995), 139-197. Zbl 0829.73010 MR 1346368

27. Tonegawa, Y. Integrality of varifolds in the singular limit of reaction-diffusion equations. Hiroshima Math. J. 33 (2003), 323-341. Zbl 1059.35061 MR 2040901

28. White, B. The size of the singular set in mean curvature flow of mean-convex sets. J. Amer. Math. Soc. 13 (2000), 665-695. Zbl 0961.53039 MR 1758759

29. White, B. A local regularity theorem for mean cuvature flow. Ann. of Math. (2) 161 (2005), 1487-1519. Zbl 1091.53045 MR 2180405

30. ZIEMER, W. P. Weakly Differentiable Functions. Springer (1989). Zbl 0692.46022 MR 1014685 